\author{
(Aus der Universitäts-Frauenklinik [weil. A. v. Rosthorn] \\ in Wien.)
}

\title{
Die Prognose von Schwangerschaft, Geburt und Wochenbett bei Herzfehlern.
}

\author{
Von \\ Dr. Rud. Th. Jaschke, \\ z. Zt. Assistent an der Königl. Frauenklinik in Greifswald.
}

(Hierzu Tafel X.)

Probleme, die ein Grenzgebiet zwischen Geburtshülfe und der Gesammtmedicin betreffen, zeichnen sich im Allgemeinen dadurch aus, dass sie kometengleich da und dort einmal auftauchen, eine kurze Spanne Zeit weitere Kreise unter den Fachcollegen interessiren und, ohne an der Wurzel gefasst zu sein, wieder in Vergessenheit gerathen. Consequent eine derartige Frage bis zu ihrer Lösung zu verfolgen, zeigt Niemand Lust. Das Grenzgebiet gilt als undankbar und ist es thatsächlich, wenn nicht Vertreter beider Gebiete Hand in Hand arbeiten oder ein Autor die in Betracht kommenden Capitel beider Gebiete einigermaassen beherrscht.

Das gilt ganz besonders für die im Titel dieser Arbeit gestellte Frage, deren Lösbarkeit eigentlich nicht zu bezweifeln ist. Nach der Zahl einzelner Arbeiten über Herzfehler und Schwangerschaft, die schon schwer zu übersehen ist, scheint es verwunderlich, dass in dieser Kernfrage bis heute keine Einigkeit erzielt ist. Wohl haben seit Hecker auch in Deutschland namhafte Geburtshelfer und manche hervorragende Internisten ihre Erfahrung in mehr oder weniger umfangreichen und gründlichen Arbeiten niedergelegt; und alle folgenden Autoren haben von gegensätzlichen oder ähnlichen Meinungen ihrer Vorarbeiter Kenntniss genommen; aber keiner Arbeit Ergebnisse haben es bisher zu allgemeiner Anerkennung gebracht. Vielfach ist es zu scharfen Discussionen über theoretisches Detail gekommen zu einer Zeit, wo die Untersuchungsmethoden ganz unzureichend waren. Beobachtungsfehler sind mit 
Jaschke, Schwangersohaft, Geburt und Wochenbett bei Herzfehlern. 467 unterlaufen und selbst in wichtigen Grundragen der Physiologie des. Kreislaufs in der Schwangerschaft gelten noch heute die widersprechendsten Ansichten.

Darauf möchte ich aber hier nicht eingehen. Wir können unsere Frage zunächst rein praktisch angreifen, gestützt auf ein Material, das schon durch die Grösse seiner Zahlen vor manchen Fehlern schützt und Beachtung fordert. Dabei wird sich noch Gelegenheit finden, die eine oder andere theoretische Frage zu streifen.

Auch wir wählen zunächst den Weg der Statistik, um das nöthige Thatsachenmaterial zu gewinnen. Zur Verfügung stand dazu das gesammte geburtshülfliche Material der zweiten Frauenklinik von Anfang 1900'1) bis März 1910, das ich in den letzten 2 Jahren selbst beobachten konnte.

Die Zahl der Geburten in dem angegebenen Zeitraum betrug 37014; darunter fanden sich 546 Fälle mit Herzfehlern, das entspricht 1,47 pCt.

Um den Ueberblick über das Material vollständig zn gestalten, sei noch Folgendes erwähnt. Nicht mitgezählt unter diesen 546 Fällen sind 50, die in den Geburtsgeschichten nicht ais Herziehler geführt sind, trotzdem nach Aetiologie und dem percutorischen sowie auscultatorischen Befund am Herzen die Diagnose zu stellen gewesen wäre, und zwar $44 \mathrm{mal}$ auf Insuff. valv. mitr., $6 \mathrm{mal}$ auf Insuff. und Stenos. valv. mitr. Der dadurch bedingte Fehler ist nicht gross. Rechnet man diese 50 Fälle mit, dann ist die Procentzahl der Herzfehler 1,6.

Wie diese 546 Fälle sich auf die einzelnen Herzfehler vertheilen, die Zabl der I p. und Multiparae, die Gesammtgeburtenzahl in jeder Kategorie von Herzfehlern, habe ich in nachstehender Tabelle (S. 468 u. 469) übersichtlich zusammengestellt. Diesellue bedarf zunächst keiner weiteren Erlüuterung.

Nach dem Alter gruppirt, betrug die Zahl der Frauen:

$$
\begin{aligned}
& \text { unter } 20 \text { Jahren . . . } 38 \quad 40-45 \text { Jahren . . . } 29 \\
& 20-29 n \text {. . . . } 302 \text { nicht notirt . . . } 29 \\
& 30-39 n \cdot .146
\end{aligned}
$$

In 42 Fällen ist der Stand nicht notirt.

1) Vor 1900 findet sich in den Geburtsgeschichten keine Rubrik für Herzbefund; es schien mil daher unthunlich, weiter zurückzugehen, da ohne solchen Zwang zur Herzuntersuchung wohl auch manches leichtere Vitium übersehen worden sein mag. 


\begin{tabular}{|c|c|c|c|c|c|}
\hline Art des Herzfehlers & I p. & II p. & III $p$. & IV p. & V p. \\
\hline $\begin{array}{l}\text { Insuff. mitral. } \\
\text { Insuff. + Sten. mitral. } \\
\text { Stenosis mitral. } \\
\text { Insuff. + }\end{array}$ & $\begin{array}{r}135 \\
31 \\
14 \\
2 \\
1 \\
- \\
- \\
1 \\
- \\
-\end{array}$ & $\begin{array}{r}96 \\
22 \\
9 \\
1 \\
4 \\
-1 \\
- \\
- \\
- \\
11\end{array}$ & $\begin{array}{l}33 \\
9 \\
4 \\
2 \\
- \\
- \\
- \\
- \\
- \\
\end{array}$ & $\begin{array}{r}25 \\
10 \\
1 \\
- \\
-1 \\
- \\
- \\
- \\
- \\
1\end{array}$ & $\begin{array}{r}16 \\
9 \\
2 \\
3 \\
- \\
= \\
= \\
- \\
- \\
4\end{array}$ \\
\hline $\begin{array}{r}\text { Gesammtzahl } \\
\text { Alter }\end{array}$ & $\begin{array}{c}191 \\
15-41\end{array}$ & $\begin{array}{c}144 \\
19-40\end{array}$ & $\begin{array}{c}52 \\
21-45\end{array}$ & $\begin{array}{c}42 \\
22-41\end{array}$ & $\begin{array}{c}34 \\
23-40\end{array}$ \\
\hline
\end{tabular}

Von Interesse für unsere Frage ist es, auch über die von uns nicht beobachteten, vorangegangenen Geburten möglichst viel festzustellen. Es fand sich, dass die mit Herzfehlern behafteten Frauen die stattliche Zahl von 1548 Graviditäten aufzuweisen haben; der Ausgang der Gravidität blieb in 63 . Fällen unbekannt; die betreffenden Frauen, theils Erst-, theils Mehrgebärende kamen in früheren Monaten der Gravidität an die Klinik, sei es, dass Blutungen oder Beschwerden von Seite des Herzens oder auch die nur schlecht verhüllte Absicht, eine Unterbrechung ihrer Schwangerschaft zu erreichen, sie zu uns führten. Sie wurden wegen mangelnder Indication zu einem Eingriff nach kürzerer oder längerer Beobachtung wieder entlassen und sind später zur Geburt nicht wiedergekommen.

Bei dem Rest der Franen liess sich in 8 Fällen anamnestisch sicherstellen, dass eine oder mehrere Geburten, im Ganzen 23, vor der Acquirirung des Herzfehlers stattgefunden hatten.

In allen übrigen Fällen fand $1311 \mathrm{mal}$ die Geburt zur rechten Zeit statt, $92 \mathrm{mal}$ kam es zur Frühgeburt im 7.-10. Nonat, $122 \mathrm{mal}$ zum Abort.

Nieht ganz unwichtig für uns und von allgemeinerem Interesse erscheint mir die Feststellung der Aetiologie des Klappenfehlers, die in der Mehrzahl der Fälle mit Wahrscheinlichkeit gelang. Die Aetiologie war
a) nicht festzustellen
b) einheitlich festzustellen
in 161 Fällen $=29,48$ pCt.
c) nicht einheitlich festzustellen 302 
Jaschke, Schwangerschaft, Geburt und Wochenbett bei Herzfehlern. 469

\begin{tabular}{|c|c|c|c|c|c|c|c|c|c|}
\hline VI p. & VII p. & VIII p. & IX p. & X p. & XI p. & XII p. & XVII p. & ? p. & $\begin{array}{c}\text { Gesammt- } \\
\text { zahl }\end{array}$ \\
\hline 11 & 10 & 9 & 4 & 4 & 4 & 1 & 1 & 2 & 350 \\
\hline 5 & 2 & 9 & 1 & 2 & 2 & 1 & - & - & 108 \\
\hline 4 & - & 1 & - & 1 & - & - & - & - & 36 \\
\hline- & 1 & 1 & - & - & - & - & - & - & 10 \\
\hline- & 1 & - & - & - & - & - & - & - & 6 \\
\hline- & - & - & - & - & - & - & - & - & 1 \\
\hline- & - & - & - & - & - & - & - & - & 1 \\
\hline- & - & - & 1 & - & - & - & - & - & 4 \\
\hline- & - & - & - & - & - & - & - & - & 1 \\
\hline- & - & - & - & 1 & - & - & - & $\ldots$ & 1 \\
\hline- & - & 一 & - & - & - & - & - & - & 1 \\
\hline 1 & - & 1 & - & - & 1 & - & - & 1 & 32 \\
\hline 21 & 14 & 21 & 6 & 8 & 7 & 2 & 1 & $\mathbf{3}$ & 546 \\
\hline $29-43$ & $29-43$ & $29-43$ & $32-4$ & $3-4$ & $37-41$ & $39-40$ & 41 & & \\
\hline
\end{tabular}

Diese Zahlen bedürfen einiger Erläuterungen.

Ad b) Als einheitlich feststellbar habe ich die Aetiologie in jenen Fällen bezeichnet, in denen anamnestisch eine der Infectionskrankheiten zu erheben war, die bekanntermaassen in der Aetiologie der Klappenfehler eine grosse Rolle spielen. In vielen Eällen gaben die Frauen an, von dieser Infectionskrankheit datirten ibre Herzbeschwerden, manche wussten auch durch ihren Arzt, dass sie im Gefolge einer bestimmten Krankheit einen Herzfehler acquirirt hatten. Auch wo solche bestimmte Angaben fehlen, dürfte die Annahme eines ätiologischen Zusammenhanges mit einer bestimmten Infection gerechtfertigt sein. Wissen wir doch seit Bamberger und Curschmann, dass oft längere Zeit vergeht, bis die Erscheinungen des Klappenfehlers manifest werden.

Unter den gewöhnlichen Ursachen eines Vitiums überwiegt weit die

Polyarthritis rheumatica mit 192 Fällen $=35,17$ pCt.; dann folgen Masern . . . . . . $, 57 n=10,44$,

Scharlach . . . . " $13 n=2,38 "$

Variola . . . . . $" 13 n=2,38 n$

Erysipel . . . . $" 3 n=0,55$,

$$
278 \text { Fällen }=50,92 \mathrm{pCt} \text {. }
$$

In 24 Fällen liessen sich als seltenere Ursachen feststellen:

5 mal Diphtherie; 2 mal ganz bestimmte Angabe über den ätiologischen Zusammenhang,

4 "Pneumonie, 
$470 \mathrm{Jaschke}$, Schwangerschaft, Geburt und Wochenbett bei Herzfehlern.

4 mal nur häufig wiederkehrende Anginen,

2 , Chorea,

$2 \pi$ Pericarditis,

$2 n$ Typhus,

1 ) Puerperalfieber, also septische Erkrankung,

$1 "$ Pleuritis,

1 . Bauchfellentzündung,

1 Lues.

Schliesslich gehört hierher 1 Fall von congenitaler Pulmonalstenose.

Diese Aufstellung enthält neben den bekannten zwar auch manche seltenere Ursache für Klappenfehler. Doch ist von jeder bekannt, dass sie gelegentlich einmal eine Rolle spielt. In dem Fail, der oben genannt ist, gab die Frau ganz bestimmt an, im ersten Wochenbett an Puerperalfieber mit Herzcomplication erkrankt und seitdem herzleidend zu sein.

Ad c) Unter den 83 Fällen mit nicht einheitlich feststellbarer Aetiologie habe ich diejenigen zusammengefasst, in denen mehrere der ätiologisch häufig in Betracht kommenden Infectionskrankheiten anamnestisch nachweisbar waren, ohne dass eine derselben als bestimmt mit der Entstehung des Herzfehlers im Zusammenhang stehend, angegeben wurde. Diese Fälle vertheilen sich auf:

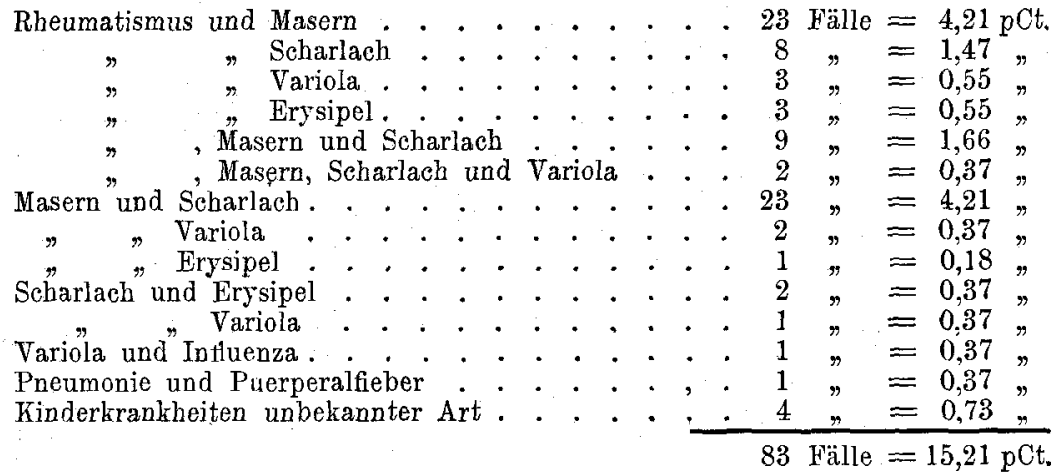

Wir können also in 44,96 pCt. der Fälle Rheumatismus nachweisen, die in der Aetiologie der Herzklappenfehler bäufigen Infectionskrankheiten in $2 / 3$ der Fälle. Für die 161 Fälle nicht feststellbarer Aetiologie ist za bedenken, dass überhaupt in nicht ganz einem Viertel der Fälle keine greifbare Ursache nachweisbar ist. „Doch dürfte sie wohl stets auf infectiöse oder toxische Einflüsse zurückzuführen sein" (Romberg, Lehrbuch S. 191). Da sich 29 unserer Frauen bereits jenseits des 40. Lebensjahres befanden, einige auch arteriosklerotische Veränderungen zeigten, 
Jaschke, Schwangerschaft, Geburt und Wochenbett bei Herzfehlern. 471

dürfte vermuthungsweise für manche Fälle wohl an einen $\mathrm{Zu}$ sammenhang mit endarteriitischen Veränderungen zu denken sein. Endlich werden wir in den Fällen, die auf Diphtherie, Typhus, Frysipel zu beziehen sind, anch auf coordinirte Muskelveränderungen unser Augenmerk richten müssen - einen prognotisch sehr ausschlaggebenden Factor. Ich möchte aber hier von einer zu weitgehenden Analyse des Materials absehen, einige einschlägige Bemerkungen können noch weiter unten vorgebracht werden.

Die bisherigen Feststellungen setzen uns in den Stand, nunmehr einer ganzen Reihe von Einzelfragen nachzugehen, nach deren Erledigung wir mit Kritik im Stande sein werden, allgemeine prognostische Gesichtspunkte zu gewinnen, die dann auch im concreten Einzelfall ihren Werth erweisen. Wir werden zu untersuchen haben:

I. Wie gestaltet sich die Mortalität?

II. Wie viele Geburten (bezw. Früh- und Fehlgeburten sind a) spontan erfolgt, b) künstlich herbeigeführt?

III. Lässt sich bei den spontanen Aborten und Frühgeburten eine Ursache finden, steht der Herzfehler damit in causalem Zusammenhiang?

IV. Was gab in den künstlich unterbrochenen Schwangerschaften die Indication zu dem Eingriff? Kritik der Indicationsstellung.

V. Verlauf der Schwangerschaft bei Herzfehler. Abhängigkeit etwaiger Beschwerden und Störungen der Schwangerschaft von dem Herzfehler. Verlauf der Schwangerschaft bei decompensirtem Vitium.

VI. Verlauf der Geburt. Gefahren, nothwendige Eingriffe, Indicationsstellung, Narkose.

VII. Verlauf des Wochenbetts.

VIIl. Lässt sich ein Einfluss der Art des Herzfehlers feststellen?

\section{Ad I.}

Es lassen sich feststellen:

a) unter 122 Fehlgeburten . . . . 1 Exitus

b) $" 92$ Frühgeburten . . . . $3 "$

c) $" 1311$ Geburten zur rechten Zeit 5 ,

also unter 1525 Geburten . . . . . 9 Exitus $=0,59 \mathrm{pCt}$.

Diese einfache Berechnung der Mortalität genügt aber nicht, um allgemein gültige Schlüsse über die Gefahren der Geburt bei 
472 Jaschke, Schwangerschaft, Geburt und Wochenbett bei Herzfehlern.

Herzfehlern zu ziehen. Es ist vielmehr unumgänglich nothwendig, die ad exitum gekommenen Fälle im Einzelnen kritisch $z u$ erörtern.

Ad a. P. No. 2772/1904. 39jührige Igrav., Mens. VI. - Seit dem 4. Graviditätsmonat, anfänglich nur wach Anstrengung, später auch ohne solche, Herzklopfen und Athemuoth. Allmähliche Steigernng dieser Beschwerden, die in letzter Zeit hohe Grade erreichen. Oedeme.

Diagnose: Sten. valv. mitr. decomp. Aetiologie: Masern.

Therapie: Wegen der zunehmenden, durch Ruhe und interne Medication nicht zu beseitigenden Decompensation Einleitung des Abortus, kurz nach dem Blasensprung Collaps und alsbald Exitus.

Obduction: Hochgradige Sten. valv. mitr.

Epikrise: Ein causaler Zusammenhang zwischen Herztod und Gravidität dürfte kaum zu bestreiten sein. Es sind aber einige Einzelbeiten zu beachten: 1. die Art des Herzfehlers. Dass die Stenose des Mitralostiums für die Erfüllung erhöhter Ansprüche besonders ungünstig ist, wird allgemein anerkannt. Die Bedingungen für den Mechanismus der Compensation sind bei diesem Klappenfehler schon an sich ungünstigere als bei irgend einem anderen. Die Gefahr der Herzerlahmung durch ungenügende Blutversorgung des Herzmuskels ist grösser. 2. Dass die Decompensation durch interne Mittel und Ruhe nicht zu beheben war, ist ein weiteres, gerade bei Mitralstenose ungünstiges Zeichen und kann bis zu einem gewissen Grade als Maass dafür angenommen werden, dass die durch die Gravidität gestellten Anforderungen an sich schon die Leistungsfähigkeit des Herzens überschritten. Der unmittelbare Beweis dafür wird durch das. Erlahmen des Herzens gegeben, sobald die Geburtsthätigkeit richtig in Gang kam. 3. ist auch das Alter der Pat. wesentlich zu berücksichtigen. Bei starker Mitralstenose ist die Chance, über das 4. Lebensjahrzehnt hinauszukommen, überhaupt gering.

Ad b. Fall 19. P.-No. 597/1907. 41jähr., XIgrav., Mens. VII, verheirathet. - 10 Geburten ganz normal, ohne Herzbeschwerden in der Schwangerschaft (Pat. giebt jedenfalls keine an). In dieser Gravidität allmählich zunehmende Herzbeschwerden. Vor 1 Monat ein Anfall hochgradiger Athemnoth mit starker Herzbeklemmung und Angstgefühl. In den letzten Wochen Herzklopfen und Athemnoth zunehmend, treten anfallsweise auf.

Diagnose: Insuff. et Stenos. valv. mitr. cum Insuff. valv. aortae. Hochgradige Decompensation, Hydrothorax. Ausserdem besteht Hydramnios. Aetiologie: Unklar.

Therapie: Einleitung der Frühgeburt. Geburt bald nach dem Blasensprung. Kurz nach der Geburt Exitus.

Obduction: Insuff. und Sten. valv. mitr. mit Verkalkung der Klappen und frischen verrucösen Auflagerungen. Insuff. valv. Aort. Fettherz, hochgradige parenchymatöse Degeneration des Herzmuskels, Hydrothorax, Blutungen im Pericard, allgemeine Stauungserscheinungen.

Epikrise: Der Fall bietet eine Menge Besonderheiten. Auffallend ist vor Allem bei dem üblen. Verlauf dieser Schwangerschaft, dass 10 vorangegangene Schwangerschaften und Geburten jedenfalls obne wesentliche Beschwerden verlaufen sind, da die Frau keine Erinnerung daran bewahrt hat. Man geht wohl nicht fehl, diese merkwürdige Thatsache mit der Art der Klappenfehler in Zusammenhang zu bringen. Es ist seit Langem bekannt - schon Traube hat darauf aufmerksam 
gemacht - dass combinirte Klappenfehler oft überraschend wenig subjective und objective Störungen hervorrufen. Weiter wissen wir, dass gerade Leute mit Aortenfehlern oft lange Zeit gar keine Beschwerden haben und selbst za grösseren körperlichen Leistungen fähig sind. Ebenso ist es hente allgemein anerkannt, dass Aortenfehler und mit solchen combinirte sonstige Herzfehler, wenn einmal eine Störung auftritt, meist eine sehr ungünstige Prognose geben. Das hängt mit dem Compensationsmechanismus zusammen, der kaum bei einem anderen Fehler so günstig ist. Aber bier kommt auch. Alles auf den Herzmuskel an. Leidet einmal seine Ernährung, dann ist der Anfang vom Ende da. Das ist meist der Ausgang der Aortenfehler. Der Process greift auf die Coronararterien über, Erscheinungen subjectiver Art deuten gewöbnlich zuerst darauf hin, dann kommt oft ganz plötzlich ein Erlahmen im Anschluss an irgend eine erhöhte Arbeitsleistung.

Diese allgemeine Schilderung passt genau auf unsern Fall. Die anfallsweise auftretenden Beklemmungen, das Angstgefühl dabei, deuten ganz klar auf eine Erkrankung der Aortenbasis (Nothnagel). Dass die recurrirende Endocarditis im Verlauf solcher Fehler nicht selten vorkommt und dann die Prognose ungünstig wird, ist ebenfalls bekannt. Die Obduction bestätigt alle diese Befunde. Sicher ist das Alter der Patienten für die Art der Veränderungen und für den Eintritt der Decompensation mit verantwortlich zu machen, und ein Theil der Veränderungen auf die Abnutzung durch die zablreichen vorhergegangenen Geburten zu beziehen. Der Tod ist ein ganz charakteristischer Tod durch plötzliche Erlahmung des Herzmuskels, der der raschen Schwankung nach den Anstrengungen der Geburt - die an sich gewiss nicht gross waren - nicht mehr gewachsen war.

Fall 571. P.-No. 1116/1908. 20jährige I grav., Mens. IX/X, verheirathet. - Seit 13 Wochen Herzbeschwerden und starke Athemnoth.

Diagnose: Insuff. und hochgradige Stenos. valv. mitralis. Schwere Decompensation (allgemeine Staung, Hydrothorax). Aetiologie: unklar.

Verlauf: 4 Wochen ante terminum spontane Frühgeburt, die von den subjectiven Beschwerden abgesehen, glatt verläuft. Am 2. Wochenbettstag Anfall heftigster Atbemnoth, starke Cyanose. Am 3. Tag sich stärker wiederholend, am 4. Tag unter zunehmender Cyanose und Athemnoth Exitus.

Obduction: Befund wie oben. Frische und alte Endocarditis.

Epikrise: Ein sicheres Urtbeil lässt sich mangels einer genaueren Anamnese über das Verhalten des Herzens vor der Gravidität und über den Eintritt der Decompensation nicht geben. Ein ungünstiger Einfluss der Schwangerschaft und Geburt scheint aber sicher zu bestehen. $O b$ für die recurrirende Endocarditis nicht eine Infection verantwortlich zu machen ist, lässt sich nicht entscheiden. Die recurrirende Endocarditis für das Versagen des Herzens verantwortlich zu machen, geht nach allgemeinen Erfahrungen nicht an. Der ganze Verlauf, sowohl die frühzeitige Decompensation in der Schwangerschaft, als auch der Tod deuten vielmehr darauf hin, dass es sich um eine recurrirende Myocarditis im Sinne der Krehl'schen Befunde bei Klappenfehlerherzen handelt, in deren Gefolge selbst eine nur mässige Steigerung der Ansprüche zu Decompensation und nach der Geburt durch Uebermüdung zum Tode führte. Sicherheit hätte hier nur die mikroskopische Untersuchung des Herzmuskels geben können. 
474 Jaschke, Schwangerschaft, Geburt und Wochenbett bei Herzfehlern.

Fall 678. P.No. 621/1910. 33 jähr. VI grav. Mens. IX/X, verheirathet. - 5 vorhergegangene Schwangerschaften und Geburten ohne Besonderheiten. Auch jetrt ungestörte Schwangerschaft ohne wesentliche Beschwerden; etwas Dyspnoe und Husten in der letzten Zeit.

Diagnose: Stenosis valv. mitral. compensata. Geringe Bronchitis. Aetiologie: unklar.

Spontane Frühgeburt (S. oben), verlänft glatt. Schon am 1. Wochenbettstag stärkere Athemnoth, herabgesetzte respiratorischeVerschieblich keit der Lungenränder. In den folgenden Tagen unter zunehroender Athemnoth Auftreten einer Dämpfung, die in die Herzdämpfung übergeht; am 4. Tag ziemlich plötzlich Exitus.

Obduction: Sten. valv. mitr. - Fitrige fibrinöse Pericarditis, Stauung in allen Organen, Hydrothorax, Ascites, etwas Hypoplasie.

Epikrise: Dass hier die Pericarditis, bes. deren Art für den ungünstigen Ausgang verantwortlich zu machen ist, dürfte keinem Zweifel unterliegen. Das Vitium spielt sicher nur eine secundäre Rolle. Die Aetiologie der Pericarditis jst uns freilich unklar geblieben. Der Herzfehler an sich, obwohl eine Mitralstenose, hat die Schwangerschaften und Geburten sämtlich gut ertragen.

Ad c. Fall 254, P.-No, 549/1905. 31 jähr. III p. ledig. - Die beiden vorangegangenen Schwangerschaften und Geburten ohne Störung, ebenso diese Schwangerschaft.

Diagnose: Insuff. et Sten. valv. mitr. compensata. Aetiologie: mit 17 Jahren Gelenkrheumatismus.

Verlauf: Bei der Geburt schwere Eklampsie. Wendung nach Braxton Hicks, Manualhilfe. Nach dem 3. eklamptischen Anfall unter Lungenödem Exitus.

Obduction: Frische endokardiale Auflagerungen, sonst conform den klinischen Erhebungen.

Epikrise: Einfluss der Schwangerschaften und Geburten auf das Herz gleich Null. Todesursache zweifellos die Eklampsie. Ob für den raschen Tod das Herz mitverantwortlich zu machen ist, kann dahingestellt bleiben.

Fall 405, P.-No. 2745/1903. 20jähr. I p. ledig. - Seit 3 Wochen etwas Husten, sonst Schwangerschaft beschwerdefrei.

Diagnose: Insuff. et Stenos. valv. mitr. e. Insuff. valv. Aortae. Tbc. pulmon. (apic.) obsoleta. Aetiologie: mit 16 Jahren Gelenkrheumatismus.

Verlauf: Geburt anfangs ohne Belang. Dann, noch in der Eröffnungsperiode nach dem Aufrichten im Bett plötzlich Herzangst, Athemnoth und in wenigen Minuten Exitus. Sectio caesarea in mortua mit lebendem Kind.

Obduction: Alte Endocarditis der Mitralis und der 1. Vorhofswand mit Schrumpfung und Verdickung der Klappen, alte Endocarditis der Aortenklappen, die ebenfalls slark verdickt und geschrumpft sind. Schrumpfung, Verdickung und Verwachsung der Sehnenfäden an der Tricuspidalis, feinste, frische thrombotische Auflagerungen, hochgradige excentrische Hypertrophie, fettige Degeneration des Myokards. Staung und Oedem in den Lungen, Schwiele nach Tuberculose in der Lungenspitze, Staung und fettige Degeneration der Nieren, Ascites, Hydrothorax.

Epikrise: Die Anstrengungen der Geburt sind für den Exitus wohl 
Jaschke, Schwangerschaft, Geburt und Wochenbett bei Herzfehlern. 475

verantwortlich. Der Fall zeigt aber soviele Besonderheiten in der Häufung und Scbwere der einzelnen Veränderungen an den Klappen, eine Miterkrankung des Herzmuskels, dass er dadurch an allgemeiner Bedeutung für die Entscheidung unserer Frage einbüsst. Denn Niemand wird imstande sein, die schweren Veränderungen an den Klappen mit der Gravidität in irgend einen causalen Zusammenhang zu bringen.

Fall 415. P.-No. 3232/1903. 30jähr. II p., ledig. - Die erste Schwangerschaft endete im 2. Monat mit Abort (vor 10 Jahren).

Diagnose: Insuff. et Stenos. valv. mitr. compensata. Aetiologie: als Kind Masern, vor 10 Jahren Gelenkrbeumatismus; vor 2 Jahren Lues.

Verlauf: Schwangerschaft und Geburt ungestört. Placentalösung. Im Anschluss daran schwere Sepsis mit Meningitis - Exitus.

Obduction: zur alten eine frische Endocarditis.

Epikrise: Der Fall scheidet aus. Ein Zusammenhang zwischen Sepsis und Herzfehler besteht nicht, Schwangerschaft und Geburt blieben durch den Herzfehler unbeeinflusst.

Fall 605. P.-No. 2707/1908. 29 jähr. I p., verh. -

Diagnose: Insuff. et Stenos. valv. mitr., Nephritis chronica in graviditate. - Aetiologie: Typhus, Gelenkrheumatismus.

Verlauf: Die Frau kommt mit schwerster Decompensation in die Klinik. Sie kann nur sitzen, nicht liegen, schlät infolge hochgradiger Atembeklemmung fast nicht. Blutdruck stark erhöht. Ein Versuch, durch interne Therapie den Zustand zu bessern, bleibt erfolglos. Da an den maximal oedematösen Beinen starke Lymphorrhoe auftritt und die Frau unter der Athemnoth und Schlaflosigkeit sebr leidet, nach 2 tägiger Beobachtung, 2 Wochen ante terminum in Narkose Hysterotomia anterior. - Lebendes Kind. Starke Atonie, Anämie. - Im Wochenbett, ausgehend von einem Ulcus puerperale am Damm, Sepsis, die am 34. Tag p. part. zum Tode führt.

Obduction: Thrombophlebitis suppurativa beiderseits, l. Parametritis. Zur alten eine frische Endocarditis, Thrombose im r. Herzohr, hämorrhagische Infarcte in zahlreichen Organen.

Epikrise: Maassgebend für die Decompeesation in der Schwangerschaft ist hier wohl die schwere Nephritis. Nephritis in der Schwangerschaft bei gleichzeitigem Herzfehler ist überhaupt eine der gefährlichsten Complicationen. Andererseits beweist der Fall, wie unglaublich viel selbst einem mit einem klappenfehler behafteten Herzen zugemuthet werden kann, wenn der Muskel intact ist. Wieweit der tötdliche Verlauf des Puerperalfiebers durch die während derselben entstandene Endocarditis verschuldet ist, lässt sich schwer sagen. Jedenfalls erscheint, allgemein gesprochen, nach meinen Erfahrungen ${ }^{3}$ ) bei nicht intactem Herzen die Prognose des Puerperalfiebers von vornherein getrübt und auch die Localisation am Herzen (sog. septische Endocarditis) durch das vorher bestehende Vitium jedenfalls in die Wege geleitet.

Fall 650. P.-No. 2251/1909. 35jähr. IIIp., verheiratet. - Zwei Schwangerschaften und Geburten ungestört.

Diagnose: Stenosis valv. mitral. decompensata. Aetiologie: Gelenkrheumatismus mit Recidiven.

1) Jaschke, Zeitschr. f. Geb. u. Gyn. Bd. LXVI. H. 2. 1910. 
476 Jaschke, Schwangerschaft, Geburt und Wochenbett bei Herzfehlern.

Verlauf: In dieser Schwangerschaft von Anfang an mehr Athemnoth, Steigerung derselben mit dem Fortschritt der Gravidität. Jetzt wird die Frau gebärend eingeliefert mit starker Cyanose, hochgradiger Dyspnoe, beginnendem Lungenödem, allgemeinen Stauungserscheinungen, schlechtem, kleinem, unregelmässigem Puls. Trotz Coffeïn und Digalen fortschreitendes Lungenödem, sodass in oberflächlichster Aethernarkose nach rascher Erweiterung mit dem zugfesten Ballon die Wendung und Extraction ausgeführt wird. Schon während der Operation wird die Athmung terminal, der Puls unzählbar, kurz nach der Entwicklung des Kindes Exitus.

Obduction: Stenosis valv. mitr., Dilatation, Degeneration des Myocards, Ascites, Hydrothorax, allgemeine Stauungserscheinungen.

Epikrise: Mangels einer genaueren Anamnese lässt sich über den Zustand des Herzens vor dieser Gravidität nichts sagen. Jetzt scheint ein ungünstiger Einfluss desselben ausser Zweifel. Die Art des Vitiums und besonders die schweren Veränderungen des Herzmuskels sind aber in Betracht zu ziehen. Ich verweise diesbezüglich auf das oben, S. 472 Angeführte. Auch das Alter der Pat. ist in Rechnung zu ziehen.

Eine kritische Uebersicht über die 9 Todesfälle führt zu folgendem Ergebniss: drei von den Todesfällen sind hier auszuschalten (Fall 678, 254,415. ), da die zum Tode führende Erkrankung mit dem Herzfehler in keinem durch dieSchwangerschaft oder Geburt bedingten causalen Zusammenhang steht, der Herzfehler bei dem ungünstigen Ausgange mindestens keine andere Rolle spielt, als ausserhalb der Gravidität. Bis zu einem gewissen Grade gilt das auch von Fall 605. Die Mortalität unter 1525 Geburten stellt sich also, je nachdem wir nur die drei erstgenannten oder auch den Fall 605 abrechnen, auf 0,39 . bezw. 0,32 pCt. Man sieht, dass nach der einfachen Mortalitätsberechnung die Gefahr für das Leben ausserordentlich gering ist. Ja, ich glaube, dieser Eindruck wird noch verstärkt, wenn wir uns einmal um die Procentzahl garnicht kümmern, sondern die Eigenart dieser sämmtlichen Fälle in Beziehung setzen zu dem, was allgemein über die Prognose von Herzfehlern bekannt ist.

Wir haben unter unseren Fällen 3 reine Mitralstenosen, ferner einen Fall, in dem die Stenose die Insufficienz weit übertraf. In 2 Fällen bestand neben hochgradigen Veränderungen der Bicuspidalklappen eine Aorteninsufficienz mit schweren Veränderungen an den Klappen. In dem zweiten dieser Fälle waren auch an der Tricuspidalklappe schwere Veränderungen vorhanden, während in dem ersten endarteriitische Veränderungen mit consecutiver Ernährungsstörung des Herzmuskels sich fanden. Auch im Falle 571 müssen wir auf Muskelveränderungen wohl ein Hauptgewicht legen, die im Fall 650 noch stärker hervortreten.

Beachtenswerth ist das Alter der Patientinnen. Nur zwei 
Jaschke, Schwangerschaft, Geburt und Wochenbett bei Herzfehlern. 477

Zwanzigjährige finden sich darunter, beide Iparae, beide mit sehr schweren Veränderungen an der Mitralis, bezw. an allen Klappen. Eine dritte Ipara mit 29 Jahren (Fall 605) kommt zunächst wegen der Nephritis und Sepsis nicht in Betracht. Alle übrigen Frauen sind im 4., eine sogar im 5. Lebensdecennium. Eine 39 jährige Ipara geht an Mitralstenose hohen Grades zu Grunde. Eine IIpara und eine III para (Meningitis, Eklampsie) scheiden hier aus. Danach bleiben noch eine III para mit 35 Jahren, eine VIpara und eine XIpara. Letztere zeigte arteriosklerotische Veränderungen mit davon abhängiger Herzmuskelinsufficienz.

Für die Prognose der Klappenfehler gilt nun auch heute noch als oberstes Gesetz: „Nicht für einen bestimmten Klappenfehler, nur für einen bestimmten Menschen kann sie gegeben werden." [v. Jürgensen $\left.\left.{ }^{x}\right)\right]$. Dazu gehört vor Allem genaue, genügend lange Beobachtung des Einzelfalles, zu der uns Geburtshelfern gewöhnlich die Möglichkeit fehlt. Wir müssen uns also damit bescheiden, den individuellen Eigenthümlichkeiten des Einzelfalles so gut als möglich Rechnung zu tragen. Da kommt zunächst die Grösse des vorhandenen Klappenfehlers in Betracht ${ }^{2}$ ), die auch wir zum Theil aus der Anamnese, insbesondere der allgemeinen Leistungsfähigkeit der betreffenden Patientin, aus dem Verhalten des Herzens bei vorhergegangenen Schwangerschaften und Geburten einigermaassen, im Zusammenhang mit dem objectiven Befund, abschätzen können. Hier machen sich dann auch gewisse Unterschiede geltend, insofern der Compensationsmechanismus bei den einzelnen Klappenfehlern ein verschiedener ist und je nach den vorzugsweise daran betheiligten Herzabschnitten auch die Aussichten auf die Dauer der Compensation verschiedene sind. Dass unter sonst gleichen Verhältnissen, bei gleicher Constitution im 3. und 4. Lebensdecennium eine Aorteninsufficienz im allgemeinen bessere Aussichten giebt, als eine Mitralstenose, ist leicht verständlich. Dieses Verhältniss kann sich aber sofort verschieben, wenn bei dem ersten Klappenfehler etwa durch wiederholte Anfälle von Endocarditis die Mitralklappen miterkranken oder an der Aorta arteriosklerotische Processe auftreten, die Neigung zeigen, auf die Kranzarterien überzugreifen und damit die Blutversorgung des Herzens zu sebädigen.

1) v.Jürgensen (Nothnagel's Handbuch XV): Klappenfehler, S.42. 1903.

2) cf. Näheres in den interessanten Ausfübrungen von Benno Lewy: Die Arbeit des gesunden und kranken Herzens. (Zeitschr. f. klinische Med. 1897. Bd. 31.) 
478 Jaschke, Schwangerschaft, Geburt und Wochenbett bei Herzfehlern.

Ebenso ist es bekannt, dass bei combinirten Mitralfehlern ein stärkeres Ueberwiegen der Stenose als wenig günstig gilt. Mitralstenosen führen schon an sich zu abnorm hohem Druck in den Lungenvenen und diese Erschwerung des Lungenkreislaufs wird durch die Schwangerschaft in den letzten Monaten sicher noch gesteigert. Damit steigen erneut die Ansprüche an das rechte Herz. Sind nun die durch die Grösse des Klappenfehlers an das rechte Herz gestellten Anforderungen schon an sich gross, dann wird leicht verständlich, dass die Steigerung der Ansprüche durch die Schwangerschaft gelegentlich an die letzte Grenze der Leistungsfähigkeit führt und eine selbst geringe Mehrforderung unter der Geburt zum plötzlichen Versagen führt. Ich meine also, bei der Mitralstenose spielt unter den uns interessirenden Verhältnissen die Grösse des Defectes eine wichtige, unter Umständen ausscblaggebende Rolle.

In erster Linie aber wird immer wieder das Hauptgewicht für die Prognose auf das Verhalten des Herzmuskels zu richten sein. Und da nun nach Krehl die Ursache des Klappenfehlers häufig auch zu Muskelveränderungen führt, so wird neben Art und Grösse des Klappenfehlers auf die anamnestische Feststellung früherer Anzeichen von Insufficienz des Herzens besonderes Augenmerk zu richten sein. Namentlich der Verlauf früherer Schwangerschaften kann werthvolle Anhaltspunkte liefern. Das einmal insufficient gewesene Herz bleibt entschieden weniger widerstandsfähig, und allgemein ist die Lebensdauer des hypertrophischen Herzens eine geringere. Die Zahl der vorangegangenen Geburten ebenso wie das Alter der Patientin müssen stets in Rechnung gezogen werden.

Ich denke, diese Bemerkungen werden genügen, die oben angeführten Fälle besser in ihrer Eigenart würdigen zu können.

\section{Ad II.}

Unter der Gesammtzahl von 1525 in ihrem ganzen Verlaufe bekannten Schwangerschaften erfolgte die Geburt

$$
\begin{aligned}
& \text { spontan } 1584 \mathrm{mal} . . . . . .=97,31 \text { pCt. u. zw. } \\
& 1306 " \text { à terme. . . . }=85,64 \text { " } \\
& 79 \text { " als Frühgeburt . }=5,18 \text { " } \\
& 99, \text { als Abort' } 1 \text { ) . . }=6,49 \text { " }
\end{aligned}
$$

1) Unter Abort habe ich sowohl die FehIgeburt im engeren Sinne, als anch den Partus immaturus gerechnet. 
Jaschke, Schwangerschaft, Geburt und Wochenbett bei Herzfehlern. 479

künstlich eingeleitet in $41 \mathrm{Fällen} \mathrm{.} \mathrm{.}=2,69$ pCt. u. $\mathrm{zW}$.

$$
\begin{aligned}
1 \text { mal à terme . . } & =0,06 " \\
17 " \text { als Frühgeburt } & =1,12 " \\
23 " \text { "Abort . } & =1,51 "
\end{aligned}
$$

Diese Feststellungen sind in mancher Hinsicht interessant; beweisen sie doch zunächst, dass in $7 / 8$ aller Fälle eine Geburt am normalen Ende der Gravidität erfolgt. Die Zahl der Aborte and Frühgeburten ist nach dieser Berechnung jedenfalls geringer als nach den Angaben der Autoren bei Herzfehlern zu erwarten wäre. Rechnen doch viele, so v. Leyden, v. Guérard, Schell ${ }^{1}$ ) und Andere bei Herzfehlern $40-50$ pCt. vorzeitige Unterbrechungen der Schwangerschaft. Demelin giebt 2,4 pCt. Aborte, $14 \mathrm{pCt}$. Frühgeburten bei Herzfehlern an, während man durchschnittlich bei allen Gebärenden $15-20$ pCt. Aborte zu rechnen hat. Nur Vinay ${ }^{2}$ ), der allerdings im Gegensatz zu den anderen Autoren das Herz jeder Schwangeren principiell untersuchte, fand unter 29 Herzfehlern nur 2 Frühgeburten $(=3,4$ pCt.), also eine unserem Ergebniss viel näherstehende Zahl. Die Differenz mag sich wohl aus dem kleinen, seiner Berechnung zu Grunde liegenden Material erklären. Als Ursache der angeblich so grossen Zahl von Schwangerschaftsunterbrechung bei Herzfehlern werden zum Theil Hämorrhagien in die Decidua und Veränderungen der Placenta, zum Theil - bei schlecht compensirten Herzfehlern - ungenügende Arterialisation des foetalen Blutes in Folge Stase in den uteroplacentaren Gefässen angeführt. Multiparae sind scheinbar öfters betroffen als Primiparae, was allerdings allgemein für Aborte angegeben wird.

Ich glaube, bei der Differenz der Meinungen verlohnt es sich, auch dieser Frage nachzugehen.

\section{Ad III.}

Jeden einzelnen Fall hier anzuführen und soweit als möglich die Genese des Abortus oder der Frühgeburt zu ergründen, würde zu viel Raum erfordern. Ich habe diese Untersuchung durchgeführt, gebe aber hier nur einige wenige Fälle, die mir besonders erwähnenswerth scheinen; das, was an allgemeinen Thatsachen sich feststellen liess, werde ich dann kurz zusammenfassen.

1) Cit. nach L. Seitz in v. Winckel's Handbuch. Ba. II. 2.

2) Vinay, Maladies du coeur et grossesse. Arch. de tocol. et de gynécol. T. XX. p. 301 . 
480 Jaschke, Schwangerschaft, Geburt und Wochenbett bei Herzfehlern.

Was zunächst die Aborte anlangt, so ist hier die Verwerthung anamnestischer Angaben eine ausserordentlich unsichere, weil wir in der Grossstadt mit einer grossen Zahl crimineller Aborte zu rechnen haben.

a) Da sind einmal 17 Fälle mit 27 Fehlgeburten, in denen ich nicht im Stande war, auch nur mit Wahrscheinlichkeit irgendwelche Vermuthungen über die Ursache der Fehlgeburt zu gewinnen.

b) In 11 Fällen mit zusammen 18 Fehlgeburten liessen sich andere Ursachen für den Eintritt der Fehlgeburt entweder sicher feststellen oder schienen mir wenigstens viel näherliegend.

3 Fälle von Lues mit 1 Abort. Mens. III, 5 Partus immaturus. Mens. V-VI.

1 Partus immaturus M. V nach langdauernder Blutung bei Placenta praevia. Die erste Geburt war normal gewesen.

1 Fall von Retroflexio uteri gravidi, Ill gravida, bei der es in allen 3 Graviditäten zum Abortus kam. (Dass die Retroflexio eine sehr häufige Ursache für Fehlgeburten darstellt, wird allgemein zugegeben. Ahlfeld behauptet sogar, dass sie der Lues den Rang streitig mache.)

1 Abortus bei einer II grav. (erste Geburt normal) im Anschluss an schwere Influenza. Die Influenza führt nach v. Engeli) häufiger als irgend eine Infectionskrankheit zum Abort. Ich erinnere ferner daran, dass Stumpf und Sperling im Anschluss an die grosse Influenzaepidemie 1889/90 sogar eine erhebliche Herabsetzung der Geburtenziffer feststellen konnten, die sie mit den durch die Influenza bedingten Schwangerschaftsunterbrechungen in Zusammenhang bringen.

1 Fall (Il grav.) mit im 1. Wochenbett erworbener Retroflexio (cf. oben).

1 sichergestellter crimineller Abort bei einer Vgravida.

1 Abort bei Uterus bicornis (in den folgenden Graviditäten Frübgeburten).

1 Fall (Vgr.) mit 2 Aborten in der 1. und 2. Gravidität, dann ein macerirtes Kind, dann 2 normale Geburten. Lues ist hier wohl wahrscheinlicher.

Ferner noch 2 Fälle von Abort, die beide höchstwahrscheinlich criminell eingeleitet waren.

Von Seiten des Herzens, meist uncomplicirte Mitralinsufficienzen, waren in allen diesen Fällen keinerlei Beschwerden und Störungen nachzuweisen.

c) Der Herzfehler scheint mir in 16 Fällen mit 38 Fehlgeburten mit grösster Wahrscheinlichkeit die Ursache oder wenigstens Theilursache für den Eintritt des Abortus.

Unter diesen Fällen sind 4 decompensirte Herzfebler, 2 Mitralstenosen. Bei einer grösseren Zahl derselben kam es wiederholt zu geringen Blutungen, ehe die Unterbrechung eintrat. Das sind die Fälle von Blutungen in die Decidua. Bei einem Theil der Fälle ist es später dann zu Frühgeburten, bei einigen nach diesen auch noch zu normalen

1) v. Engel, Wiener med. Presse. 1896. No. 43 und 44. 
Geburten gekommen. Mehrere dieser Fälle werde ich noch unter den Frühgeburten und unter IV, anzuführen haben und sebe deshalb hier von einer näheren Erörterung derselben ab.

d) Endlich sind noch zu erwähnen 15 Fälle mit 16 Aborten, in denen unter einer grösseren Zahl von Schwangerschaften nur 1 Abortus vorkam, vorher und nachher wieder normale Geburten erfolgten und keine Störung der Compensation sich fand. In diesen Fällen scheint mir die causale Bedeutung des Herzfehlers ganz unsicher. Ich persönlich möchte sie wenigstens nicht durch das Herzleiden herbeigeführt erachten.

Wir haben also von 99 spontanen Abortus, bezw. Partus immaturus etwa 34 Fälle als nicht durch den Herzfehler bedingt, auszuscheiden. Danach würde die Zahl der Spontanaborte 4,2 pCt. ausmachen. Weiter zu gehen in Deutung unserer Beobachtungen and anamnestischer Daten scheint mir aus den oben angegebenen Gründen unsicher.

Unter 79 spontanen Frühgeburten sind 45 bloss anamnestisch festgestellt, 44 von uns selbst beobachtete. Bei der ersten Gruppe ist bezüglich der Ursache über blosse Vermuthungen nicht hinauszukommen.

In dem schon oben erwähnten Falle von Uterus bicornis scheint die Missbildung für die vorzeitige Unterbrechung der Gravidität verantwortlich zu machen.

In 4 Fällen dürfte die Schwere des complicirten Vitiums und die Decompensation für Aborte und Frühgeburten verantwortlich zu machen sein. Davon wird einer noch weiter unten $z u$ besprechen sein.

Zwei andere Fälle zeigten wiederholt in der Schwangerschaft geringe Blutungen. Eine $\mathrm{V}_{\mathrm{p}}$, eine $\mathrm{XI}$. In beiden Fällen war es auch wiederholt zum Abort gekommen.

In 4 Fällen, sämmtlich Ilp. mit compensirten Mitralinsufficienzen, war es beide Male zu Frühgeburten gekommen.

Bei einer III para mit compensirter Mitralinsufficienz und starker Kyphoskoliose trat jedesmal die Frähgeburt ein. Die Raumbeengung des Herzens dürfte hier eine wesentliche Rolle gespielthaben. Fs ist bekannt, dass ein hypertrophisches Klappenfehlerherz dạfür empfindlich ist.

In 2 Fällen trat unter 7 bezw. 8 Graviditäten nor einmal eine Frühgeburt (einmal nur um 2 Wochen zu früh) ein, so dass der Einfluss des Vitiums wohl zweifelhaft erscheint.

In 22 Fällen endlich vermag ich gar keine Vermuthungen aufzustellen. Zweimal ist die Art des Herzfehlers nicht näher verzeichnet, in allen übrigen handelte es sich um compensirte Mitralinsufficienzen, die unter einer $\mathrm{Zahl}$ von 3-8. Graviditaten je eine Frühgeburt aufzuweisen hatten.

Etwas mehr liess sich bei den 34 unter unseren Augen sich abspielenden Frühgeburten feststellen. 
482 Jaschke, Schwangerschaft, Geburt und Wochenbett bei Herafehlern.

Da sind zunachst eine Vpara, eine Xpara und eine XIpara za nennen, die unter allen Graviditaten nur diese eine Frühgeburt hatten, dabei uncomplicirte und vollständig compensirte Mitralinsufficienzen aufwiesen. Ferner eine II para, bei der die Geburt kaum 2 Wochen zu früh eintrat.

Lues ist als Ursache der Frühgeburt in 4 Fälen nachweisbar oder mindestens sehr wahrscheinlich.

In 3 Fällen dürite begleitende sehwere Nephritis die Ursache für die Frühgeburt sein; dabei war dieselbe in einem Falle wohl auch für die vorbandene Decompensation verantwortlich zu machen.

In einem Fall trat die Frähgeburt im Anschluss an fieberhafte Endocarditis (Rheumatismusrecidiv) ein.

Eine Frühgeburt, die wir beobachteten, fällt auf den schon oben erwähnten Fall von Uterus bicornis.

In 19 Fällen scheint mir der Herzfehler für die Frühgeburt verantwortlich. Vier davon betrafen decompensirte Herzfebler. Bei neun Fällen unter diesen war es bei früheren Graviditäten zum Abort, dann Partus immaturus, dann Frühgeburt zu einem früheren Termin gekomuen.

Mit diesen kurzen Daten muss ich mich aus Raumrücksichten begnügen. Zusammenfassend lassen unsere Beobachtungen etwa folgende Schlüsse zu:

Nach Ausscheidung von 7 Fällen, in denen die Frühgeburt sicher auf andere Ursachen (Lues, Missbildung, Kyphoskoliose) za beziehen ist, ferner einer XIpara und XIIpara, die unter sonst ganz normalen Schwangerschaften und Geburten nur diese eine Frühgeburt aufzuweisen hatten, stellt sich die Gesammtzahl spontaner Frügeburten bei Herzfehlern auf 4,5 pCt. Diese Zahl ist aber noch zu hoch, da die mit Nephritis complicirten Fälle und eine Frühgeburt im Anschluss an Infectionskrankheit darin einbezogen sind. Es kann sich ja aber nur um einen Annäherungswerth handeln. Im Durchschnitt werden $4-5 \mathrm{pCt}$. das Richtige treffen.

Ein besonderes Hervortreten der Frühgeburt je nach der Art des Herzfehlers lässt sich an diesem Material nicht feststellen. Decompensation scheint aber entschjeden zur Frühgeburt zu disponiren, wobei wohl die Stase in den uteroplacentaren Gefässen eine Rolle spielen mag. Die Frühgeburt kann in solchen Fällen als eine Art Selbsthilfe der Natur aufgefasst werden, was auch für unser therapeutisches Handeln bei nicht zu behebender Decompensation Fingerzeige giebt. Die allgemeine Beobachtung, dass höhere Geburtenzahl und höheres Alter zur Frühgeburt mehr disponiren, gilt auch bei Herzfehlern. 
Jaschke, Schwangerschaft, Geburt nnd Wochenbett bei Herzfeblern. 483

Von einer Verschlimmerung des Herzleidens ist in den wenigen in dieser Kategorie in Betracht kommenden Fällen nichts zu constatiren. Besonders auffallend scheint aber die Thatsache, dass in einer relativ grossen Zahl der Graviditäten die Tragzeit der betreffenden Frauen eine immer steigende Dauer zeigt, eine Thatsache, auf die meines Wissens bisher nur Baranger ${ }^{1}$ ) hingewiesen hat. $\mathrm{Ob}$ es berechtigt ist, in seinem Sinne von einer "accoutumance aux cardiopathies" zu sprechen, müsste erst durch noch grössere derartige Beobachtungsroihen erwiesen werden.

Alles in Allem genommen, ist also sicher zuzugeben, dass Herzfehler, namentlich wenn sie decompensirt oder mit anderen Krankheiten complicirt sind, Veranlassung zur Frühgeburt geben können, doch darf diese Gefahr nicht überschätzt werden, da sie auf 20-25 Frauen mit Herzfehlern höchstens einmal zutrifft.

\section{Ad IV.}

Dass bedrohliche Zustände bei Herzfehlern eine Indication zur Einleitung der Frühgeburt abgeben, wird heute allgemein anerkannt. Um in das Wesen unseres Problems einzudringen, müssen wir daher auch alle jene Fälle berücksichtigen, in denen die Schwangerschaft künstlich unterbrochen wurde. Eine weitere Aufgabe wird darin bestehen, mit sorgfältiger Kritik die Indicationsstellung in den einzelnen Fällen zu prüfen. Es wird vor Allem festzustellen sein, $o b$ dieselbe in allen Fällen eine strenge war, jnwiefern sie unseren erweiterten Kenntnissen und Erfahrungen entspricht. Ebenso wird der Erfolg des Eingriffes in Betracht gezogen werden müssen. Die Resultate dieser Untersuchung werden wir dann verwerthen, um allgemeine Gesichtspunkte für die Indicationsstellung zu gewinnen, und dieselben auch für die Prognose mit Nutzen verwerthen können.

Es sind im ganzen 41 Fälle, die wir hier zu berücksichtigen haben (cf. oben, Seite 479).

a) Einleitung der Geburt: 1 Fall.

1. Fall 531. P.-No. 2023/1900. 26jährig, I para, ledig. Schwangerschaft ungestört bis auf Oedeme seit 3 Monaten.

Diagnose: Insuff. valv. mitral. Aetiologie: unbekannt.

Verlauf: Einleitung der Geburt, Forceps.

1) Baranger, Cardiopathies et grossesse. Thèse de Paris. 1898. 
484. Jaschke, Schwangerschaft, Geburt und Wochenbett bei Herzfehlern.

Kritik: Da von Seiten des Herzens gar keine Störungen vorhanden waren, nicht einmal die Oedeme mit Sicherheit darauf zu beziehen sind, ist der Eingriff indicationslos.

b) Einleitung der Frülgeburt: 17 Fälle.

2. Fall 19. cf. Seite 472. Kritik: Berechtigt.

3. Fall 37. P.-No. 1473/1907. 38jährig, V para, Mens. VII, verheirathet. - Die ersten drei Schwangerschaften und Geburten ohne Besonderheiten, in der vierten Schwangerschaft starke Oedeme bereits in den ersten Monaten, vermehrte Palpitationen, im 5. Monat Spontanabort. Ueberhaupt seit 5 Jahren stärkere Herzbeschwerden.

Diagnose: Insuff. und Stenos. valv. mitral. mit Insuff. valv. tricuspid., letztere als Folge der Decompensation. Aetiologie: Mit 18 Jahren Gelenkrheumatismus.

Verlauf: Ausbildung der Decompensation schon um die Mitte der Schwangerschaft, jetzt zunehmende Verschlechterung. Daher Einleitung der Frühgeburt. Starke Nachblutung. Auftreten von Herzschwäche, die durch Digalen beboben wird. Danach allmähliche und fortschreitende Besserung.

Kritik: Nach dem thatsächlichen Befunde muss die Berechtigung zur Untersucbung anerkannt werden. Vermisst wird aber ein Versuch interner Medication. Möglicher Weise wäre dadurch die Einleitung der Frühgeburt überhaupt unnöthig geworden oder hätte bis zur Erzielung eines lebensfäbigen Kindes aufgeschoben werden können.

4. Fall 69. P.-No. 2335/1907. V grav., Mens. VIII, verheirathet. - Die ersten 3 Schwangerschaften und Geburten ohne Besonderheiten, die vierte endete mit Spontanabort. Subjective Beschwerden zunehmend.

Diagnose: Insuff. valv. mitral. mit Insuff. tricuspid, letztere wieder Folge der hochgradigen Decompensation, Stauungserscheinungen in allen Organen.

Verlauf: Wegen der Decompensation. Einleitung der Frühgeburt. Glatter Verlauf.

Kritik: Wie in Fall 3. In beiden Fällen deutet aber der glatte Verlauf von 3 Sehwangerschaften, die Unterbrechung der vierten unter Zunahme der Herzbeschwerden auf eine Verschlimmerung des Herzleidens, die durch den Verlauf der fünften Schwangerschaft (Eintritt der Decompensation) offenkundig wird. Das Alter ist dabei mit in Rechnung zu ziehen

5. Fall 325. P.-No. 1358/1904. IV grav.

6. Fall 378, P.-No. 62/1903. II grav.

7. Fall 411. P.-No. 1428/1903. Il grav.

Kritik: Diese 3 Fälle, sämmtlich jüngere Frauen mit vollständig compensirten Mitralinsufficienzen scheiden hier aus, da in allen Graviditäten die Frühgeburt lediglich wegen engen Beckens eingeleitet wurde.

8. Fall 447. P.-No. 960/1902. 42jährig, VIII grav., Mens. IX/X, verwittwet.

9. Fall 462. P. No. 2671/1902. 26jährig, II grav., Mens. X, ledig.

Kritik: Die beiden Fälle scheiden hier ebenfalls aus. Die Indication zur Einleitung der Frähgeburt 3 bezw. 2 Wochen ante terminum grav, wurde thier durch scliwere Nephritis gegeben. Das Herz - in beiden Fällen Mitralinsufficienz - vermochte seine Arbeit relativ sehr 
Jaschke, Schwangerschaft, Geburt und Wochenbett bei Herzfehlern, 485

gut zu leisten; ausser Kurzathmigkeit traten von dieser Seite keine Beschwerden auf. ledig ${ }^{1}$ ).

10. Fall 479. P.-No. 44/1901. 34jährig, IIIgrav., Mens. VIII,

Diagnose: Insuff. und Stenos. valv. mitral. decompensata. Aetiologie: Rheumatismus.

Verlauf: Wegen gleichzeitigen Hydramnios', Oedemen, starker Dyspnoe, die sich schliesslich za Orthopnoe steigert, Einleitung der Frühgeburt. Compensation nach derselben erst ganz allmäblich zurückkehrend.

Kritik: Der Eingriff ist berechtigt. Ob zwischen Hydramnios und decompensirtem Vitium ein causaler Zusammenhang besteht, ist schwer zu sagen. Angenommen wird das wohl. Wäre umgekehrt die Decompensation von der enormen Leibesausdehnung abhängig, so würde der Fall dadurch eine Sonderstellung einnebmen.

11. Fall 496. P.-No. 2233/1901. 30jährige IV grav., Mens. VIl., verheirathet.

Diagnose: Insuff. und Stenos. valv. mitral. decomp. mit Insuff. valv. tricusp. Aetiologie: Gelenkrheumatismus.

Verlauf und Kritik: Da die Decompensation durch interne Therapie fast gar nicht gebessert wird, berechtigter Weise Einleitung der Frühgeburt. Nach derselben Besserung.

12. Fall 529. P.-No. 1850/1900. 34jähr., Vgr., Vens. VIIl/IX, verbeirathet. Schon gegen Ende der beiden letzten Graviditäten stärkere Kurzathmi:keit.

Diagnose: Insuff. et Sten. valv. mitr. decompensata.

Aetiologie: Gelenkrheumatismus.

Verlauf: Athemnoth bis zu Orthopnoe sich steigernd, bedrohliches Aussehen, Einleitung. Im Wochenbett unter Herztherapie Besserung.

Kritik: Wie in Fall 3 und 4.

13. Fall 540. P.-No. 3001/1900. 27jähr., Igr., Mens. X, ledig. Der Fall scheidet aus. Bei compensirter Mitralinsufficienz wurde wegen Pyelitis die Frühgeburt eingeleitet.

14. Fall 592. P.-No. 1986/1908. 31jähr., Vlp., ledig. Auszuscheiden; gleich Fall 5-7.

15. Fall 600. P.-No. 2415/1900. 31jühr., VIgr., Mens. VII, verheirathet.

Diagnose: Insuff. et Stenos. valv. mitr. mit leichten Stauungserscheinungen. Ausserdem Phthisis pulmon. et laryngis.

Kritik: Die Indication zur Schwangerschaftsunterbrechung wird nicht durch das Vitium an sich, sondern durch das Zusammentreffen mit Kehlkopftuberculose gegeben erachtet. Diese Indicationsstellung muss als berechtigt anerkannt werden.

16. Fall 605; cf. S. 475 .

17. Fall 627. P.-No. 280/1909. 35jähr., X grav., Mens. VIII/IX, verheirathet. In der 9 . Schwangerschaft, vom 5. Monat ab, stärkeres

1) Wo über den Verlauf früherer Schwangerschaften oder Geburten nichts erwähnt ist, sind dieselben als völlig normal verlaufen anzusehen. Der Kürze halber führe ich nur das Allernothwendigsto an. So habe joh auch die Decompensationsbeschwerden nirgends angegeben und nur besonders auf fällige Erscheinungen der Compensationsstörung namentlich erwähnt. 
486 Jaschke, Schwangerschaft, Geburt und Wochenbett bei Herzfehlern.

Herzklopfen, zunebmende Kurzathmigkeit; nach der Geburt Besserung. Jetzt bald nach Beginn der Gravidität zunehmende Herzbeschwerden, 5 Ohnmachtsanfälle.

Diagnose: Insuff. et Sten. valv. mitr. decompensata. Aetiologie: Diphtherie.

Kritik: Da unter Digitalistherapie keine Besserung eintritt, Herzklopfen, Athemnoth und besonders Schlaflosigkeit die Patientin quälen, ferner ein weiterer Ohnmachtsanfall auftritt, und auch mit Rücksicht auf die Zahl der Graviditäten ist die Unterbrechung vollkommen be. rechtigt.

18. Fall. P.-No. 17/1907. 37jähr., Vgr., Mens. VII, verheirathet. Seit der 4. Gravidität zunehmende Beschwerden.

Diagnose: Insuff. et Sten. valv. mitr. decompensata.

Verlauf und Kritik: Wie in Fall 17.

c) Einleitung des Abortus: 23 Fälle.

19-25. Sieben Fälle, fünf compensirte Mitralinsufficienzen und zwei Mitralstenosen scheiden hier aus, da die Fehlgeburt wegen progredienter Lungentuberculose, einmal wegen Nephritis bei mangelhaft compensirter Mitralstenose eiuer VIIIgrav, eingeleitet wurde, also aus anderer Indication

26. Fall 38. P.-No. 1562/1907. 29jähr, Igr., Mens. VI, verheirathet. Masern.

Diagnose: Insuft. et Sten. valv. mitr. decompensata. Aetiologie:

Kritjk: Da Digitalistherapie ohne Erfolg ist, Einleitung des Partus immat. berechtigt, wie auch die nachher auftretende Besserung zeigt.

27. Fall 47. P.-No. 1624/1907. 32 jährige IV grav. mens. VI. Drei Geburten vor dem Herzfehler.

Diagno se: Insuff. et Sten. valv. mitr. decompensata et. Insuff. valv. tricuspid. Aetiologie: Gelenkrheumatismus.

Kritik: Soweit nach der Geburtsgeschichte möglich, gleich Fall 3.

28. Fall 215. P.-No. 2836/1906. 39 jähr. X grav. mens. II., verh. Die achte und neunte Schwangerschaft endeten mit Abort. Seit der letzten Schwangerschaft, vor 3 Jahren, Herzklopfen und Kurzathmigkeit, die in der Gravidität jetzt zunebmen.

Diagnose: Insuff. valv, mitr.

Verlauf: Auf strikte Indicationsstellung seitens des Internisten trotz Compensation Einleitung des Abortus.

29. Fall 359 . ef. S. 472 , ad a.

30. Fall 430. P.-No. 2542/1903. 40 jähr. IV grav. mens. VI, verheirathet. Seit der letzten Schwangerschaft und Geburt, vor 8 Jahren, häufigeres Herzklopfen; vor 3 Jahren soll ein Recidiv der Endocarditis erfolgt sein. Seit Beginn dieser Gravidität immer mehr sich steigernde Herzbeschwerden:

Diagnose: Insuff. et Stenos. valv. mitr. decompensata (auch mässiger Ascites). Aetiologie: Gelenkrheumatismus.

Verlauf und Kritik: Nach dem künstlichen Abortus Besserung. Die Schwangerschaftsunterbrechung mit Rücksicht auf den objectiven Befund, Anamnese und Alter gerechtfertigt. Es bleibt aber auch hier die Frage offen, ob bei zweckmässiger interner Therapie und Schonung die Unterbrechung nicht bis zur 35: oder 36 . Woche hätte aufgeschoben werden können. 
31. Fall $419_{2}$. P.-No. 3363/1903. 29 jähr. VII grav. mens. IV/V, verheirathet. Schon in den beiden letzten Schwangerschaften Druck in der Herzgegend, Angstzustände, die erst Monate nach der Geburt gut wurden. Jetzt gesellt sich dazu stärkeres Herzklopfen, Oedeme, grosse Mattigkeit.

Verlauf: Nach Angabe des Hausarztes hat der Herzfehler unter dem Einfluss der letzten Graviditäten sich wesentlich verschlechtert. Auf sein Drängen, da der Geburt nur mit grosser Sorge entgegengesehen werden könne, wird der Abortus eingeleitet.

Diagnose: Insuff, valv. mitr. compensata. Aetiologie: unklar.

Kritik: Nach dem objectiven Befunde ist trotz der Angaben des Hausarztes die Schwangerschaftsunterbrechung. mindestews zu diesem frühen Termin unberechtigt. Die Frau hätte allerdings mit Rücksicht auf die zweifellose ungünstige Beeinflussung durch die Gravidität in in Beobachtung bleiben müssen. Auch wäre nach Auftreten eventueller Decompensation mit Rücksicht auf Anamnese und Geburtenzahl mit einer Unterbrechung sicherlich nicht lange $\mathrm{zu}$ warten gewesen. Wichtiger. wäre es wohl gewesen, neue Graviditäten durch die operative Sterilisirung unbedingt zu verhüten, besonders mit Rücksicht auf das noch jugendliche Alter der Patientin. Unter allen Umständen bestand zu einem dringenden Vorgehen keine Indication und bätte nur eigene Beobachtung und Functionsprüfung die Entscheidung geben dürfen. 32. Fall 478. P.-No. 1985/1902. 30 jühr. II grav. m. VI., verheiratet.

Diaguose: Insuff. et Sten. valv. mitr. decompensata.

Kritik: Da auf Digitalistherapie die Decompensatiou nicht zurückgeht, ist die Schwangerschaftsunterbrechung berechtigt. Nach derselben zunächst kaum Besserung.

33. Fall 542. P.-No. 3364/1900. 30 jähr. I grav. mens. V, ledig.

Diagnose: Insuff. et Stenos. valv. mitral. compensata. Epilepsie.

Kritik: Als Indication zur Schwangerschaftsunterbrechung wird einfach der Herzfehler angegeben. Diese Indicationsstellung kann nicht als berechtigt angesehen werden.

34. Fall 573. P.-No. 1183/1908. 36 jähr. VIII grav., verheirathet.

Diagnose: Insuff. et Stenos, valv. mitral. compensata. Apicitis obsoleta.

Verlauf: Der consultirte Internist hält die Stenose an sich für eine Indication zur Schwangerschaftsunterbrechung, die darauf vorgenommen wird. ledig.

35. Fall 576. P.-No. 1285/1808. 28 jähr. II grav. m. IV,

Diagnose: Insuff. et Sten. valv. mitr. decompensata.

Verlanf: Auf Rath des Internisten Schwangerschaftsunterbrechung, trotzdem unter Digitalistherapie die Decompensation prompt zurückgeht.

36. Fall 594. P.-No. 2088/1908. 37 jühr. VIII grav. m. III, verheirathet. Erste bis dritte Schwangerschaft und Geburt. Dann soll zweimal Spontanabort im 3. Monat erfolgt sein, in der 6. und 7. Gravidität wegen des Herzfehlers die künstliche Unterbrechung vorgenommen worden sein. Jetzt Mangel jeder Compensationsstörung; die Frau hegt aber den dringenden Wunsch, von ihrer Gravidität befreit zu werden. Unentbunden entlassen.

Diagnose: Insuff. valv. mitr. compensata. Aetiologie: Gelenkrheumatismus. 
488 Jaschke, Schwangerschaft, Geburt und Wochenbett bei Herzfehlern.

Kritik: Mangels näherer Angaben und der zweifelhaften Wahrhaftigkeit der Fran ist eine Kritik der damaligen Indicationsstellung unmöglich.

37. Fall 636. P.-No. 802/1909. 25 jähr. II grav. mens. II, verheiratet. Bei der ersten Geburt starke Nachblutung. Jetzt Herzklopfen, Kurzathmigkeit.

Diagnose: Insuff. et Sten. valv. mitral., an der Grenze der Compensation.

Kritik: Unberechtigt. Nähere Angaben über die Gründe fehlen.

38. Fall 643. P.-No. 1340/1909. 34 jähr. III grav, mens. II/III, verheirathet. - 2. Geburt Abort. arteficialis.

Diagnose: Insuff. et Stenos. valv. mitral. et Insuff. valv. aortae decompensala. Aetiologie: Variola, wiederholt recidivirender Gelenkrheumatismus, mit 27 Jahren Nephritis.

Kritik: Da der Internist gegen 1907 (2. Schwangerschaft) eine beträchliche Verschlimmerung, vor allem Beteiligung des Herzmuskels constatirt, ist die Unterbrechung vollständig gerechtfertigt. Das Alter und die Art des Herzfehlers sind dabei mit in Betracht zu ziehen. (cf. S. 472.)

39. Fall 641. P.-No. 1229/1909. 33 jähr. II gr. m. VII, ledig. Vor 2 Jahren von anderer Seite im 6. Monat der Gravidität wegen des Herzfehlers Einleitung des Abortus. Jetzt kommt die Frau mit Herzklopfen, Kurzathmigkeit, verminderter Harnmenge zu uns. Unter Digitalisbehandlung innerhalb weniger Tage Rücklkehr zur Norm. Die Frau wird völlig beschwerdefrei entlassen.

Diagnose: Insuff. et Stenos. valv. mitr. compensata. Aetiologie: Gelenkrheumatismus mit 2 Recidiven.

Kritik der Schwangerschaftsunterbrechung vor 2 Jahren nicht möglich. Jetzt besteht dazu keine Indication.

40. Fall 675. P.-No. 326/1910. 29 jähr. VIII grav. mens. V, verheirathet. Erste bis dritte Schwangerschaft und Geburt ohne Besonderheiten. In der vierten Schwangerschaft starkes Herzklopfen. Wäbrend der folgenden Schwangerschaften erreichen die Herzbeschwerden allmählich immer höhere Grade. Sämtliche Geburten à terme. In dieser Schwangerschaft von Anfang an stärkeres Herzklopfen, Schwindel, Ohrensausen, sodass Pat. schliesslich ihren Haushalt mit den zahlreichen Kindern nicht mehr besorgen kann.

Diagnose: Insuff. et Stenos. valv. mitral. Aetiologie: Zahlreiche Anginen.

Verlauf: Da ausser geringer Cyanose, respiratorischer Irregularität der Herzaction (Puls 77) und Kurzathmigkeit bei Körperanstrengung keine Abnormität besteht, jede sonstige Stauungserscheinung fehlt und die Functionsprüfung relativ rasche Frholung ergiebt, wird zunächst abgewartet. Unter Digitalisbehandlung wird die Frau so gebessert, duss sie auch bei leichter Körperarbeit sich ganz wohl befindet und daher zunächst entlassell.

Sie bleibt in Beobachtung. Bereits nach 8 Tagen stellen sich unter dem Einfluss häuslicher Sorgen und der Betreuung der zahlreichen Kinder etc. wieder subjective Beschwerden ein, die allmählich immer mehr zunehmen. Objectiv ist stärkere Cyanose und Kurzathmigkeit nacbzuweisen. Unter neuerlicher Digitalisbehandlung in der Klinik schwinden alle Beschwerden. Sobald die Frau in ibren häuslichen Sorgen- und Arbeitskreis zurückkehrt, das alte Spiel. 
Schonen kann sich die Frau nicht. So wird schliessilich mit Rücksicht auf die zahlreichen Geburten (sämtliche Kinder leben), die rasche Aufeinanderfolge der Graviditäten und die zweifellose Verschlechterung des Herzleidens unter dem Einfluss der letzteu Geburten, endlich unter Berücksichtigung der sozialen Lage (in Abwesenbeit der. Frau muss der Mann bei den Kindern bleiben und verliert dadurch seinen Verdienst), die eine Schonung ausschliesst, der Abortus eingeleitet, einige Wochen später die Frau sterilisirt.

Kritik: Nach der besonderen Lage des Falles ist die Schwangerschaftsunterbrechung berechtigt. Der Fall zeigt klar, welche bedeutsame Rolle die soziale Lage unter Umständen spielen kann. In solehen Fällen müssen auch solche Momente in der Indications. stellung berücksichtigt werden.

41. Fall 682. P.-No. 350/1910. 43jähr. VIgrav., Mens. V, verbeirathet. - In den beiden letzten Schwangerschaften zunehmende Herzbeschwerden. Der Hausarzt schickt die Frau zur Unterbrechung.

Diagnose: Stenos. valv. mitral. comp.

Verlauf: Zunächst Entlassung. Nach 3 Wochen kommt die Frau, die jetzt auch psychisch ganz auf die Schwangerschaftsunterbrechung eingestellt ist, wieder, mit Klagen über Herzklopfen, Gefübl von Schwere auf der Brust, Schlaflosigkeit und Husten. Objectiv ist ausser Staunngsbronchitis keine Compensationsstörung nachweisbar.

Da die Bronchitis unter Digitalisbehandlung sich nicht bessert und unter Berücksichtigung eines dahingeheuden Rathes des Internisten, wird der Abortus eingeleitet. Objectives und subjectives Befinden 3 Wochen nach demselben in Nichts gebessert.

Kritik: Die Berechtigung der Schwangerschaftsunterbrechung ist zwar nicht ohne Weiteres zu bestreiten, besonders mit Rücksicht auf Alter, Zahl der Geburten, Art des Herzfehlers und die zweifellose Verschlechterung unter dem Einfluss der letzten Graviditäten und des Alters. Trotzdem scleint mir die Indicationsstellung nachträglich zu wenig streng. Besserung wurde nicht erzielt, Gefahr bestand keine, also hätte man ruhig bis zur Erzielung eines lebensfähigen Kindes warten sollen. Die Anforderungen der Gravidität hatten sich bis dahin nicht als zu boch erwiesen, die Verschlechterung ist mehr allgemeinen Ursachen zuzuschreiben, vor allem wohl dem Alter. Wichtiger scheint mir auch bier, weitere Schwangersebaften zu verhüten.

Ueberblicken wir nunmehr die Gesammtheit der Fälle, so ergiebt sich, dass wir 23 Fälle auszuscheiden haben, darunter 14, in denen die Schwangerschaft aus ganz anderer Indication unterbrochen wurde. Warum in den Fällen 1, 31, 33 und 37 die Schwangerschaftsunterbrechung für unberechtigt gehalten wird, habe ich schon kurz erwähnt. Subjective Beschwerden oder die blosse Thatsache, dass ein Herzfehler vorhanden ist, dürfen niemals eine Indication zur Einleitung der Fehloder Frühgeburt abgeben. Wohl giebt es sebr quälende subjective Symptome, worunter in erster Linie die manchmal durch nichts zu bekämpfende Schlaflosigkeit gehört. Dieselbe kommt 
490 Jaschke, Schwangerschaft, Geburt und Wochenbett bei Herafehlern.

aber nur bei decompensirten Klappenfehlern vor. Bei einem compensirten Vitium wird selten über Schlaflosigkeit geklagt und dann ist sie durch ein- oder mehrmalige Darreichung eines Hypnoticums leicht zu bekämpfen. Bei decompensirten Herzfehlern dagegen berubt sie auf der Blutüberfüllung des Gehirns und der Hirnhäute und kann meist erst mit der Beseitigung der Decompensation behoben werden.

Selbst die Autoren, welche die Complication der Schwangerschaft mit Herzfehler für sehr ungünstig erklären, sehen bei Comsation keine Indication zur Schwangerschaftsunterbrechung. Nur v. Guérard ${ }^{1}$ ), dessen Angst vor Herzfehlern wohl Niemand theilt, geht soweit, bei Multiparen auch ohne Compensationsstörung die Schwangerschaftsunterbrechung zu empfehlen, wenn bei einer früheren Geburt gefährliche Situationen aufgetreten sind. Ich glaube, das geht zu weit. Es kann gewiss im einzelnen Fall dadurch einmal die Indication erleichtert werden, aber principiell darf man nicht derartige Sätze aufstellen. Uebrigens zeigt die Erfahrung an unserem grossen Material, dass die Geburt bei compensirten Herzfehlern eigentlich nie zu unüberwindlicher Gefahr führt.

Auch in den Fällen 28, 34 und 35 möchte ich nach den geburtshülflichen Erfahrungen die Indicationsstellung für eine zu ängstliche und darum unberechtigte halten. Die Differenz in der Auffassung der Gefahren der Geburt einerseits, des Herzfehlers andererseits bedingt hier die verschiedene Indicationsstellung. Dass die hohe Geburtenzahl und das Alter der Patientinnen, ferner die in den letzten Jahren zweifellose Verschlechterung des Zustandes des Herzens eine Ueberanstrengung als gefährlich erscheinen lassen, ist zweifellos richtig. Die Geburt als gefährliche Ueberanstrengung. ist es aber, die dem Internisten bei solcher Indicationsstellung vorschwebt, wenn auch nur die geringsten Anzeichen dafür vorhanden sind, dass ein Klappenfehlerherz über den Höhepunkt seiner Leistungsfähigkeit und Lebensdauer hinaus ist. Das habe jch selbst früher geglaubt, das war auch der Gedankengang des berathenden Internisten, wie ich aus mündlichen Auseinandersetzungen seither erfahren habe. An der logischen Consequenz dieses internistischen Gedankenganges und seiner Basirung auf allgemeiner Erfahrung ist auch nichts zu deuteln. Nur als Geburtshelfer haben wir hier einen Irrthum aufzuklären. Einmal kommen die Anstrengungen der

1) v. Guérard, Monatsschrift Bd. XII und Festschrift für Martin. 1895. 
Jaschke, Schwangerschaft, Geburt und Wochenbett bei Herzfehlern. 491

Geburt nicht plötzlich, unvorbereitet an das Herz heran. Sondern in langer Zeit, in der die Anforderungen ganz allmählich, kaum merklich von Woche zu Woche wachsen, wird das Herz an immer grössere Arbeitsleistung, an Raumbeschränkung u. s. w. gewöhnt, wie in einer systematischen Uebungstherapie. Das Plus an Arbeitsleistung bei der Geburt wird dann, wie unsere geburtshülfliche Erfahrung an so grossem Materiale lehrt, fast immer gut vertragen. Das Gefährliche der Geburtsarbeit für das Herz liegt nach unserer Erfahrung durchaus nicht in einer Ueberanstrengung, sondern in dem jähen Wechsel der Druckverhältnisse gegen Ende der Austreibungsperiode. Anders möchte die Sache sein, wenn die Geburtsarbeit plötzlich ohne vorhergegangene Uebung durch die Schwangerschaft dem Herzen zugemuthet würde. Natürlich ist immerhin ein gewisses Maass von Leistungsfähigkeit des Herzmuskels noch nothwendig. Und ich wüsste nicht, was uns berechtigte, einem Herzen dieselbe abzusprechen, ehe noch Erscheinungen von Decompensation sich gezeigt haben. Gerade beim Klappenfehler sind wir ja in dieser Hinsicht in einer günstigeren Situation: wir haben im Allgemeinen - wenn arteriosklerotische Processe mit Uebergreifen auf die Coronariae ausgeschlossen werden können kaum jemals beim Herzfehler mit einem plötzlichen Erlahmen zu rechnen, das nicht mehr zu beheben wäre. Wie ein warnendes Signal stellt sich vielmehr vorher die Herzinsufficienz allmählich ein. Bleibt also in der Schwangerschaft die Decompensation aus, dann dürfen wir das im Allgemeinen auch als ein Zeichen dafür nehmen, dass die zur Geburt erforderliche Leistungsfähigkeit noch vorhanden ist. Stellen sich Decompensationserscheinungen ein, dann ist die Ueberlegung schon schwieriger. Hier wird man nicht mehr in jedem Falle sagen dürfen: Lässt sich die Compensation wieder herstellen, dann kann zugewartet werden. In diesem Falle sind vielmehr alle die einzelnen Nebenumstände des betreffenden Einzelfalles, wie Alter, sociale Verhältnisse, Verlauf der früheren Schwangerschaften, insbesondere alle auf Verschlechterung der Herzkraft durch die Geburten hindeutenden Momente, genauestens in Betracht zu ziehen. In den beiden ersten unserer Fälle (28 und 34) würde ich beim Auftreten von Decompensation, selbst wenn dieselbe zu beheben gewesen wäre, auch nicht zu lange mit der Schwangerschaftsunterbrechung gezögert haben. Anders dagegen in Fall 35. Trotzdem hier zunächst Decompensationserscheinungen geringen Grades vorhanden 
492 Jaschke, Schwangersehaft, Geburt und Wochenbett bei Herzfehlern.

waren, scheint mir doch das prompte Zurückgehen derselben auf Digitalisgebrauch auf einen recht guten Zustand des Herzmuskels schliessen zu lassen. Derselben Ansicht sind die Internisten auch; es ist wieder nur eine übertriebene und - sagen wir es offen ganz falsche Vorstellung der Geburtsvorgänge, die das Unbekannte als so gefährlich erscheinen lässt. Gewiss möchte ich eine derartige Frau bei der Geburt keiner Hebamme überlassen. Die Austreibungsperiode bedarf ganz sicherlich genauer Ueberwachung. Wo irgend Zweifel an der Leistungsfähigkeit des Herzmuskels auftauchen, muss natürlich jede medicamentöse Hülfe zur Bekämpfung acuter Herzschwäche bereitstehen, noch wichtiger aber wird es sein, dafür zu sorgen, dass die Aufregung der Kreissenden möglichst auf ein Minimum reducirt, jeder allzu rasche Fruchtwasserabfluss (nöthigenfalls durch Kolpeuryse) vermieden wird und der Durchtritt des Kindes so schonend als möglich und langsam erfolgt. Wo diese Forderungen in derartigen Fällen sich ohne weiters nicht erfüllen lassen, scheint mir eine leichte Halbnarkose das richtige Vorgehen. Dem Glauben, dass Herzfehlerkranke durch die Narkose besonders gefährdet seien, möchte ich auch hier neuerlich entgegentreten, eine einwandfreie Narkosetechnik natürlich vorausgesetzt ${ }^{1}$ ). Die Entbindung ist dann vielfach zweckmässig durch Forceps zu bewerkstelligen, dabei aber nach der Geburt des Kopfes das Kind ganz langsam zu entwickeln. Nach dem Durchtritt des Kindes möchte ich in solchen Fällen sehr eine Bandagirung des Abdomens und das Auflegen von Sandsäcken für die ersten 6-12 Stunden empfehlen.

Vielfach scheint sich in der Literatur auch der Irrthum eingeschlichen zu haben, als bedeutete die Schwangerschaftsunterbrechung gewissermaassen ein Heilmittel, als würde nach Wegschaffung des Eies auch die durch die Schwangerschaft event. hervorgerufene Schädigung des Herzens beseitigt. Cessante causa cessat effectus. Ja, eine weitere Schädigung durch die Schwangerschaft fällt freilich weg und in vielen Fällen kann damit auch dauernder Gewinn erzielt werden. Ein "aber" jedoch hinkt nach, Einmal beweisen verschiedene Fälle (unter den oben genannten z. B. besonders Fall 41) dass, sobald die Schädigung des Herzens einmal einen gewissen Grad erreicht hat, der Wegfall der Schwangerschaft gar keine Aenderung hervorruft, woraus mir auch

1) Näheres cf. Therapeut. Monatshefte. 1909. Heft 1 u. 2. 
Jaschke, Schwangerschaft, Geburt und Wochenbett bei Herzfehlern. 493

hervorzugehen scheint, dass die Schwangerschaftsunterbrechung nicht immer der causa cessanda entspricht. Solche Fälle zeigen doch klar, dass die Schädigung noch in anderen Momenten zu suchen ist und wir gehen nicht fehl, das Alter oder ein Fortschreiben des Processes an den Klappen, bezw. Ernährungsstörungen im Herzmuskel als solche Momente anzusehen.

Damit soll aber keineswegs behauptet werden, dass der Schwangerschaft kein schädigender Einfluss zukommt. Allerdings scheint mir, als hätten in jugendlichem Alter, bei nicht zu schweren Klappenveränderungen und Fehlen jeder schwereren Muskelschädigung eine oder zwei, häufig selbst drei Schwangerschaften so gut wie nichts zu bedeuten. Die Zahl variirt natürlich ganz nach der Lage des einzelnen Falles. Das Schädigende ist meiner Ansicht nach einzig und allein in einer grösseren Zahl von Schwangerschaften und Geburten zu suchen, namentlich dann, wenn dieselben rasch aufeinanderfolgen. Schon an der völlig gesunden Frau hinterlässt die in kurzer Zeit sich häufende Geburtenzahl, die folgende Sorge um eine grössere Kinderschar, das körperliche Plus an Arbeit deutlich seine Spuren, so dass sie früh welkt. Verständlich genug, dass diese Spuren sich bei der Kranken tiefer eingraben und das kranke Organ sich zuerst dagegen auflehnt. Diese Thatsache ist schon früheren Autoren an kleinerem Material aufgefallen, ich kann sie an unseren Fällen durchwegs verfolgen, wenn ich auch finde, dass häufig, bei leichteren Herzfehlern und von kräftigen Frauen, 6 , ja selbst 7 und 8 Sehwangerschaften ertragen werden, ehe ein ungünstiger Einfluss sich bemerkbar macht, an dem dann oft genug auch das Alter, um die Wende des vierten Lebensdecenniums, seinen Einfluss hat.

Treten Complicationen auf (cf. Fall 15), unter welchen ich ganz besonders die Nephritis und die mit Kehlkopftuberculose verbundene Lungenphthise hervorhebe, dann scheint mir die Indication viel eher zu stellen, mit therapeutischen Versuchen nicht zu viel Zeit zu verlieren zu sein.

Und noch ein Punkt ist hervorzuheben: die Frühgeburt, namentlich nach dem achten Monat, einzuleiten, dünkt mich nur dann berechtigt, wenn eine absolute dringende Indication vorliegt. Geringere Strenge in der Indicationsstellung, zu welcher mir gerade in dieser Frage manchmal Internisten zu neigen scheinen, beruht auf einer ganz falschen Einschätzung der Frühgeburt gegenüber der Geburt. Es ist ein Irrthum, zu glauben, dass eine künst- 
494 Jaschke, Schwangerschaft, Geburt und Wochenbett bei Herzfehlern.

lich eingeleitete Frühgeburt an das Herz der Frau wesentlich geringere Ansprüche stellt als eine Geburt zur rechten Zeit. Die Geburtsarbeit ist fast dieselbe, zu Ungunsten der Frühgeburt fällt aber die meist längere Dauer derselben in Folge mangelhafter Wehenthätigkeit in die Wagschale, und nicht selten werden operative Eingriffe, wie Wendung und Extraction noch nachträglich nothwendig und müssen oft beschleunigt werden, wenn man nicht im letzten Moment ein lebendes und lebensfähiges Kind verlieren will.

Kehren wir zurück zu der Frage, von der wir ausgegangen sind: in wie viel Procent der Fälle war eine Schwangerschaftsunterbrechung nothwendig? so bleiben nur 18 Fälle übrig. Wir haben also nur in etwa 1 pCt. der Fälle, mit der Nothwendigkeit einer vorzeitigen Unterbrechung der Schwangerschaft zu rechnen, ein weiterer Beweis dafür, wie übertrieben die Gefahren der Herzfehler vielfach geschildert wurden.

\section{Ad V.}

Um die geburtshülfliche Bedeutung der Herzfehler richtig zu würdigen, dürfte es sich empfehlen, die Zeit der Schwangerschaft, Geburt und des Wochenbetts, jede für sich zu betrachten. Für die Schwangerschaft haben wir eine wichtige Frage schon erledigt: die Zahl der Schwangerschaftsunterbrechungen. Alle Thatsachen und Möglichkeiten, die für einen causalen Zusammenhang zwischen Herzleiden und vorzeitiger Unterbrechung der Schwangerschaft in Betracht kommen, eine Reihe von Zufälligkeiten und Irrthümern baben in den beiden vorangehenden Capiteln ihre Besprechung erfahren.

Hier bleibt uns also noch die Besprechung der Schwangerschaftsbeschwerden, soweit sie durch den Herzfehler hervorgerufen oder verstärkt sein können. Seiner prognostischen Bedeutung wegen werden wir dann noch den Verlauf der Schwangerschaft bei Decompensation etwas genauer verfolgen.

Vollständig ohne Beschwerden, die mit dem Herz in $\mathrm{Zu}-$ sammenhang sein könnten, verliefen die Schwangerschaften bei 418 Frauen $=76,5$ pCt., also über $3 / 4$ aller Frauen. (Auf die Gesammtzahl der Geburten 88 pCt.)

Von den übrigen 128 Frauen gaben

a) 39 in $\mathbf{6 4}$ Graviditäten als einzige Beschwerde Herzklopfen an. Theilweise bestanden diese Beschwerden nur während 
Jaschke, Schwangerschaft, Geburt und Wochenbett bei Herzfehlern. 495

eines Theiles, gewöhnlich in der zweiten Hälfte der Schwangerschaft, theilweise aber auch fast während der ganzen Gravidität. Diese Zahlen sind natürlich nur als ungefähre Annäherungswerthe zu betrachten. Ich bin überzeugt, dass Herzklopfen in Wirklichkeit in einem viel grösseren Procentsatz vorkommt. Leichtere Grade von Palpitation mögen der Erinnerung der Frauen, namentlich wo es sich um frühere Schwangerschaften handelt, wohl ganz entschwunden sein. - 15 dieser Erauen gaben für 20 Schwangerschaften ausdrücklich an, auch ausserhalb der Gravidität öfters Herzklopfen gehabt zu haben, jedoch in geringerem Grade.

b) Ueber Herzklopfen und Kurzathmigkeit klagten 35 Frawen in 47 Schwangerschaften. Auch diese Zahl ist auffallend gering, beweist aber immerhin, dass jedenfalls eine empfindliche Störung des Allgemeinbefindens oder der Arbeitsfähigkeit bei der grossen Mehrzahl der Herzfehler fehlt.

c) In 38 Schwangerschaften (31 Frauen) traten Zeichen von Stauung in den Lungen, Nieren, Leber, Gehirn auf, die theils vereinzelt, theils zusammen kürzere oder längere Zeit bestanden, häufig nur bei Ruhe oder unter dem Einfluss der Therapie wieder vorübergehend oder dauernd verschwanden. Auf die Art der hierdurch ausgelösten zahlreichen Beschwerden möchte ich nicht weiter eingehen. Sie sind keine anderen als ausserhalb der Gravidität. Doch scheint mir ein Punkt des Hervorhebens werth: die bei Nichtgraviden oft äusserst lebhafte Beschwerden hervorrufenden Oedeme (auch wenn sie nicht sehr hochgradig sind), die passive Hyperämie und Schwellung der Leber und Nieren geben bei Schwangeren in diesen leichteren Graden fast nie Anlass zu subjectiven Klagen. Darauf hindeutende Klagen wurden nur so nebenbei bei den Hauptklagen über Herzklopfen oder Husten vorgebracht. Husten als Zeichen einer Staungsbronchitis ist häufig eine der ersten und Hauptklagen bei Mitralfehlern, besonders bei der Mitralstenose. Ueber die Schlaflosigkeit habe ich schon oben das Wesentlichste erwähnt (S. 489ff).

d) In 20 Fällen (mit 20 Schwangerschaften) traten dieselben Symptome viel stärker hervor. Dazu gesellten sich vielfach Ascites, Hydrothorax, ausgedehnte Oedeme - kurz es handelte sich in diesen Fällen um ausgesprochene schwere Decompensation. Darunter gehören auch 6 von den Todesfällen (cf. S. $472 \mathrm{ff}$.). Ebenso haben wir die Fälle 1, 37, 38, 47, 69, 430, 478, 479, 496, 576 bereits unter IV besprochen. In den übrigen 4 Fällen handelt es sich im 
496 Jaschke, Schwangerschaft, Geburt und Wochenbett bei Herzfehlern.

Fall 97. II yrav., Mens. I/II. um Insuff. und Stenos. mitral. mit Insuff. tricusp. (Die Decompensation kann hier wohl nicht von der Schwangerschaft abhängig gemacht werden. Die Frau wurde auf die innere Klinik überwiesen.)

Fall 432. VIpara, 41jährig, mit Herzmuskelinsufficienz bei Mitralfehler. Entsprechend der Betheiligung des Herzmuskels traten Athemnoth, Beklemmung mehr anfallsweise auf.

Fall 672. IX grav, Mens. VII, 38jährig. - Decompensirte Mitralinsuff. mit Hydrothorax, Hydramnios und schwerer Nephritis, bei der es zur Frühgeburt kam.

Fall 409. V para, 34 jährig. - Insuff. und Stenose der Mitralis und Aorteninsufficienz mit hochgradigsten Oedemen, Ascites am Ende der Gravidität.

Aus dieser Zusammenstellung geht hervor, dass wir in $90 \mathrm{pCt}$. aller Fälle mit einer ohne irgend gefährliche Störung verlaufenden Schwangersehaft rechnen können. Denn die unter a) und b) genannten Beschwerden bedeuten jedenfalls keine schwere Störung und vor Allem keine Gefahr. Ausserdem zeigt sich auch hier wieder die Thatsache, dass - von besonderer Schwere oder Complicationen einzelner Fälle abgesehen — die meisten Störungen erst in späteren Graviditäten auftreten. So finden wir die unter a) genannten Beschwerden

$$
\begin{aligned}
& 8 \mathrm{mal} \text { in der ersten, } \\
& 2 n \pi n \text { zweiten } \\
& 2 n " n \text { dritten Gravidität. }
\end{aligned}
$$

Ganz ähnlich liegen die Verhältnisse in den anderen Kategorien. So kommen auch von den 20 Fäller ausgesprochener Decompensation nur 6 auf I p., 5 auf II p. und III p., eine Thatsache, die um so mehr in die Augen fällt, wenn man die Fälle einzeln betrachtet.

Dass die ausgesprochene Compensationstörung, namentlich bei älteren Frauen und Vielgebärenden, im Allgemeinen als Indication zur Schwangerschaftsunterbrechung gelten kann, haben wir schon erwähnt. In den kritischen Bemerkungen zu den einzelnen Fällen, in denen aus diesem Grunde die Fehl- oder Frühgeburt eingeleitet wurde, haben wir auch die verschiedensten Momente bereits erwähnt, die im einzelnen Fall die Indicationsstellung beeinflussen können.

Ich möchte aber diese Sätze nicht so verstanden wissen, als gäbe Decompensation an sich eine Indication zur Schwangerschaftsunterbrechung. Immerhat der Versuch interner Therapie voranzugehen. Gehen, alle Staungserscheinungen vollkommen zurück, dann wird nur unter Berücksichtigung der 
ganzen individuellen Lage des Einzelfalles eine Entscheidung fallen dürfen. Was für die Unterbrechung auch dann noch in Betracht kommen kann, babe ich an einem Falle (cf. S. $488 \mathrm{ff}$ ) schon hervorgehoben. Hier möchte ich nun einen Fall anführen, der als Gegenbeispiel dienen soll:

P.-No. 2925/1908. 22jährige Igrav., Mens. VI, verheirathet. Seit dem 9. Jahre, im Anschluss an Gelenkrheumatismus, herzleidend. Von jeher leichte Beschwerden, die seit dem Eintritt der Pubertät zunahmen. Jetzt seit 3 Wochen zunehmende Kurzathmigkeit, stärkeres Herzklopfen, Schlaflosigkeit, seit 14 Tagen kurzer Husten.

Befund am 2. 11.: Athemnoth. Pat. hatte sitzend im Bett Respiration 34, subicterisches Colorit, mässige Cyanose der Lippen, Wangen, Nase, Fingernägel. Geringes Oedem der Unterschenkel, deutlich nur in der Umgebung der Knöchel.

Herz: Heftige präcordiale Pulsation, Spitzenstoss verbreitert, hebend, bis in die vordere Axillarlinie zu fühlen. Das Herz ebenfalls verbreitert. Insuff. und Stenose der Mitralis.

Ueber den Lungen vereinzelt Rasseln, Stauungserscheinungen mässigen Grades von Seiten der Leber und Nieren, sowie der Milz.

Ueber den Verlauf giebt die Tafel X jeden Aufschluss. Die Besserung. nach den ersten 3 Tagen ist bloss auf den Einfluss der Ruhe und Milch diät zurückzuführen.

Vom 6. Tage ab ist die Pat, vollkommen frei von Beschwerden, sämmtliche Stauungserscheinungen sind verschwunden. Am 7. Tage steht sie auf, geht beschwerdelos herum und wird am 8. Tage auf ibren eigenen Wunsch nach Hause entlassen.

Das jugendliche Alter der Patientin, die ausserordentlich rasche vollkommene Beseitigung aller Stauungserscheinungen und Beschwerden, die Thatsache der ersten Gravidität und die Anamnese, die bisher noch keine Decompensation ergab, berechtigten uns wohl zu diesem Zuwarten.

Uebertriebene Aengstlichkeit ist jedenfalls auch nach den Ergebnissen dieses Abschnittes ganz unnöthig; ein Schematisieren in der Indicationsstellung soll unter allen Umständen vermieden werden.

\section{Ad VI.}

Wir kommen nunmehr zu dem kritischen Punkte unserer Erörterung. Dass die Schwangerschaft bei Herzfehlern mindestens selten zum Tode führt, haben auch andere Autoren meist zugestanden. Die Hauptgefahr sollte in der Geburtsperiode, speciell in der Austreibungszeit vorhanden sein oder es sollten doch die in dieser Zeit gesetzten Schädigungen den Tod im Wochenbett mit herbeiführen können. In wenigen wissenschaftlichen Fragen ist so viel mit Hypothesen gearbeitet worden als in dieser. Auch wir werden theoretische Streiflichter nicht ganz vermeiden können. 
498 Jaschke, Schwangerschaft, Geburt und Wochenbett bei Herzfehlern.

Zunächst aber wollen wir uns an die thatsächlichen Feststellungen halten.

Da ergiebt sich zunächst schon die auffallende Thatsache, dass von 1525 Geburten $1493=97,8$ pCt. ganz ungestört verlaufen sind. Unter ungestört soll in diesem Falle natürlich nur ungestört von Seite des Circulationsapparates verstanden werden. Demgemäss habe ich auch Fehl- und Frühgeburten hier mitrerechnet, gleichgültig auch, ob sie spontan oder auf irgend welche Eingriffe erfolgten, wenn nur die Geburtsarbeit selbst, bezw. der lieselbe etwa ersetzende Eingriff (bei eingeleiteten Aborten und sheilweise auch Frühgeburten) ohne Störung von Seiten des kranken Herzens ertragen wurde. Auch die Fälle, in denen schon in der Schwangerschaft vorhandene subjective Empfindungen von Seiten des Herzens und Kurzathmigkeit unter der Geburt zwar da, aber nicht wesentlich gesteigert waren, sind hier mit eingerechnet. Denn ich glaube, nur solche Symptome, die entweder für die Frau besonders quälend oder Zeichen von Gefahr sind, brauchen wir hier auszuschalten. Auch Abnormitäten der Geburt sind hier unberücksichtigt gelassen, wenn sie nachweislich mit dem Herzfehler in keinem Zusammenhang stehen können. So wird wohl Niemand bei engem Becken nach langer Geburtsarbeit auftretende Wehenschwäche, um nur ein Beispiel zu nennen, auf den Herzfehler beziehen wollen. Durchaus sind unter diesen ungestört verlaufenen Geburten auch solche bei schweren Herzfehlern (cf. Tabelle 1), ferner Fälle, die mit Nephritis, Tuberculose, Eklampsie complicirt waren. Ausserdem gehören hierher 10 Fälle, bei denen trotz mehr oder minder hochgradiger Decompensation die Geburt ganz glatt verlief.

Von den im Verlauf der Geburt Störungen zeigenden Fällen sind zunächst

a) 6 zum Exitus gekommene Fälle zu nennen (cf. S. $472 \mathrm{ff}$.); weiter

b) 5 Fälle, bei denen eine beträchtliche Steigerung der subjectiven Beschwerden, vor Allem Oppression, Schwächegefühl, Dyspnoe sich zeigte. Drei von diesen Frauen waren III p., eine VI p., eine VII p., drei davon waren über 30 , eine über 40 Jahre alt. Namentlich anführen möchte ich davon 3 Fälle:

1. Fall 406. 31jährige III para. - Insuff: et Sten. valv. mitral. et Insuff. valv, aort. compensata. In der Austreibungsperiode trat starke Dyspnoe, Herzoppression, Cyanose auf. Ich erinnere aber zur Würdigung dieser Erscheinungen an das bei Fall 19 Seite 472 ff Erwähnte. 
2. Fall 491. III para, 30 Jahre alt. - Insuff. und Stenos. valv. mitral. mit geringer Decompensation. Bei der im 9. Monat spontan erfolgten Frühgeburt starke Dyspnoe (64), kleiner schlechter Puls (144), Cyanose. Nach der Ausstossung des Kindes rasch Besserung.

3. Fall 648. 26jährige III para. - Stenos. valv. mitral. Bei der ersten Geburt sollen Herzkrämpfe aufgetreten sein. Diese Geburt verläuft glatt.

c) In 10 Fällen traten bedrohliche Erscheinungen auf.

1. Fall 37 , cf. Seite 484.

2: Fall 153. P.-No. 1279/1906. 28jährige Ipara. - Insuff. valv. mitral. decompensata. Aetiologie: Gelenkrheumatismus. Im Beginn der Geburt hochgradigste Athemnoth, Tachycardie. Wendung, Extraction, Perforation des nachfolgenden Kopfes (enges Becken) in CbloroformAethernarkose. Nach der Geburt Erholung.

3. Fall 173. P.-No. 1740/1906. 36 jährige VII para. - Insuff. und Stenos. valv, mitral. decompensata Aetiologie: Gelenkrheumatismus. Bereits einmal Decompensation. Trotzdem sind die früheren 6 Geburten ohne Störung verlaufen. In dieser Schwangerschaft seit zwei Monaten starke Athemnoth, Herzklopfen, die sich nach Digitalisbehandlung bessern. Jetzt tritt unter der Geburt ein starker Collaps auf. Beendigung der Geburt durch Wendung und Extraction. Dann allmähiche Erbolung unter interner Behandlung.

4. Fall 272. P.-No, 2393/1905. 41jährige X para. - Insuff. et Stenos. valv, semilun, sin. Schwangerschaft ohne Besonderheiten. Bei Hydramnios Wendung und Extraction. Unmittelbar nach der Ausstossung des Kindes Collaps, jedoch unter Kampher rasche und volle Erholung.

5. Fall 294. P.-No. 3220/1905. 40jährige V para. - Insuff. et Stenos. valv. mitral. Nephritis in graviditate. Tuberc. laryngis. Bei im 9. Monat spontan erfolgender Geburt heftigste Dyspnoe, Cyanose. Nach der Geburt Erholung. Im Wochenbett Thrombose der Saphena.

6. Fall 413. P.-No. 1527/1903. 22jährige Ilpara. - Insuff. valv. mitral. et aortae, leichte Decompensation mit Stauungserscheinungen in der Niere, Oedeme. Bei der ersten Geburt soll Herzschwäche mit beginnendem Lungenödem aufgetreten sein, so dass die Geburt beschleunigt werden musste. Jetzt glatter Verlauf.

7. Fall 432 P.-No. 2703/1903. 41jährige VIpara. - Vitium decompensatum, Myodegeneratio cordis. 5 Graviditäten und Geburten ohne Besonderheiten. Jetzt seit 3 Monaten Oedem, Herzklopfen, in letzter Zeit starke Athemnoth, seit 6 Wochen anfallsweise Dyspnoe, Gefühl vou Schwere auf der Brust, starke Beklemmung (richtiger wohl als anginoide Zustände bei chronischer Herzinsufficienz zu bezeichnen).

Kurz nach dem Wehenbeginn hochgradigste Athemnoth, die nach der Geburt des ersten Zwillings eher noch stärker wird. Das zweite Kind, in Querlage, wird gewendet und extrahirt. Dabei wird der Puls schiecht, anzählbar, Stridor tritt auf (beginnendes Lungenodem), trotzdem gar kein Narcoticum gegeben wurde. Unter Analepticis Ueberwindung der Gefahr, dann allmähliche Erholung.

8. Fall 479. P.-No. 44/1901. 34jährige III para. - Vitium decompensatum bei gleichzeitigem hochgradigen Hydramnios. Hochgradigste Dyspnoe, nach der Geburt Vitium noch längere Zeit decompensirt.

9. Fall 488. P.-No. 953/1901. 41jăhrige Ipara. - Stenos, valv. mitral. In Beginn der Geburt Puls 150, inaequal, starke Cyanose, 
Dyspnoe. Der Puls steigt auf 176, wird klein. Nach der Geburt allmähliche Erbolung.

10. Fall 519. P.-No. 3380/1901. 21 jährige I para. - Insuff. valv. aortae et mitral., complicirt mit Nephritis. Unter der Geburt Herzschwäche, beginnendes Lungenödem, unter Herzmitteln Erholung.

d) Bei 8 Frauen mit 11 Geburten trat geringere oder stärkere Atonie auf. In 2 Fällen war dieselbe geringfügig, in 8 Fällen, darunter 3 mal bei Mitralstenose, 1 mal bei Zwillingen ist dieselbe als stark verzeichnet. In einem Fall endlich kam es in Folge der Atonie zum Collaps.

Insgesammt waren also nur bei 32 Geburten $=2,2$ pCt. Störungen von Seiten des Herzens oder Abnormitäten bej der Geburt, die vom Herzen abhängig sein können, nachweisbar. Genauere Untersuchung würde aber noch eine Anzahl Fälle auszuscheiden gestatten. Zunächst unter den zum Exitus gekommenen Fällen zwei (Fall 294, 605), in denen für den Tod wohl nicht das Zusammentreffen von Herzfehler und Geburt verantwortlich gemacht werden kann. Im Fall 204 und 519 ist die Complication mit Nephritis zu berücksichtigen, Fall 432 zeigt so ausgesprochen die Symptome ungenügender Herzleistung in Folge degenerativer Vorgänge im Herzmuskel, dass er nicht eigentlich unter vom Herzfehler abhängige Gefahren fällt. - Auffallend ist weiter, dass es sich überwiegend um Mehrgebärende und vor Allem ältere Frauen handelt, ein Umstand, der gewiss für die Beurtheilung nicht gleichgültig ist; deutet er doch klar darauf hin, dass von einem Vitium auch unter der Geburt eigentlich nur dann Gefahr droht, wenn der Herzmuskel geschädigt ist, was mit der Lebensdauer des hypertrophischen Klappenfehlerherzens in engen Beziehungen steht. - Und was schliesslich die Atonien anlangt, so ist ihre Zahl an sich eine so geringe, dass man jedenfalls nach diesen Zahlen nicht behaupten kann, Herzfehler würden $\mathrm{zu}$ atonischen Blutungen disponiren. Zum Theil ist ihr Zusammenhang mit dem Herzfehler wohl mehr als fraglich.

Gleichgültig aber, wie dem auch sei, wir können praktisch jedenfalls behaupten, dass im Allgemeinen die Gefahr der Geburt bei Herzfehlern eine sehr geringe ist und bisher fast allgemein sehr überschätzt wurde.

Es ist ganz besonders die Austreibungsperiode, die als gefährlich geschildert wird, während in der Eröffnungsperiode die Gefahren geringer sein sollen. Geben einige Autoren nur allgemein als Ursache der Gefahr die zur Zeit der Presswehen auftretenden 
Jaschke, Schwangerschaft, Geburt und Wochenbett bei Herzfehlern. 501

starken Druckschwankungen im Allgemeinen an, so drücken sich Andere bestimmter aus. Essen-Möller ${ }^{1}$ ) sieht die Gefahr in der intrathoracalen Drucksteigerung und Behinderung der Aspiration in die Venen durch die forcirte Exspirationsstellung während der Presswehen, andere fürchten die plötzliche Abnahme des intraabdominalen Druckes bei rascher Entleerung von viel Fruchtwasser und besonders nach der Ausstossung des Kindes. v. Guérard2) glaubt sogar, dass auch eine gut überstandene Geburt für das Herz einen dauernden Schaden bedeute, der sich unfehlbar bei späteren Geburten bemerkbar mache. Ich halte das für ganz ausserordentlich übertrieben. Am richtigsten hat wohl Fellner ${ }^{3}$ ) die möglicher Weise gefahrdrohenden Phasen des Geburtsvorganges geschildert. Ich verweise ausdrücklich auf diese Arbeit, eine der besten in dieser ganzen Frage. Fellner hat auch die Literatur so sorgfältig zusammengestellt, dass ich von einer neuerlichen. Wiedergabe derselben absehen kann, umsomehr, als die seitdem erschienenen, meist nur kleinen casuistischen Arbeiten die Frage nicht weiter gefördert haben. Schildert Fellner auch an der Hand seiner Blutdruckversuche die Gefahren der Druckschwankungen sehr anschaulich, so kommt er nach den rein practischen Erfahrungen zu einem viel günstigeren Resultat.

Meine eigenen Untersuchungen und Erfahrungen lassen sich mit Uebergehung aller Details etwa so zusammenfassen:

Nicht die Thatsache eines vorhandenen Herzfehlers bedeutet - von seltensten Fällen schwerster Klappenveränderungen abgesehen - eine Gefahr, sondern lediglich eine der Klappenerkrankung eventuell coordinirte Herzmuskelerkrankung. Der Unterschied zwischen Schwangerschaft und Geburt ist dabei nur ein gradueller. Sind schwerere Veränderungen des Herzmuskels da, dann versagt er schon früher oder später im Verlauf der Schwangerschaft (Decompensation), kann aber auch dann noch in manchen Fällen die Mehrforderung unter der Geburt überwinden, selbst wenn es nicht gelingt, die Decompensation vorher vollständig zu beseitigen. Erträgt ein Herz aber die in der Schwangerschaft zweifellos vor-

1) Essen-Möller, Ueber Schwangerschaft und Geburt bei organischen Herzfehlern. Hygiea. Bd. 63. S. 345. 1901. ref. Monatsschrift. Bd. XV. Seite 604.

2) v. Guérard, líc.

3) Follner, 0. 0., Herz und Schwangerschaft. Monatsschr. f. Geb. und Gyn. Bd. XIV. 1901. 
502 Jaschke, Schwangerschaft, Geburt und Wochenbett bei Herzfehlern.

handene Arbeitsvermehrung, dann ist auch für die Geburt so gut wie nichts zu fürchten. Die Schwangerschaft bedeatet in solchen Fällen eine sehr sorgsam dosirte Uebungstherapie.

Die Mehrforderungen unter der Geburt sind die Belastungsprobe auf die Accommodationsbreite des hypertrophischen Herzens. Ebenso wie ein gesundes Herz erträgt auch ein gesunder hypertrophischer Herzmuskel die bei einer normalen Geburt auftretenden, z. Th. sehr rasch erfolgenden Druckschwankungen. Das ist ja gerade ein Charakteristikum des Herzmuskels, dass er innerhalb weiter Grenzen das Vielfache seiner gewöhnlichen Arbeit sofort in dem Augenblick zu leisten vermag, in dem sie an ihn herantritt ${ }^{1}$ ). Maassgebend ist dabei freilich das Training der Schwangerschaft; es besteht hier ein ganz ähnliches Verhältniss wie beim Training des Bergsteigers. Das vollkommen gesunde Herz des gelehrten Stubenhockers ist nicht ohne weiteres im Stande, etwa die zur Ersteigung des Montblanc nothwendige Arbeit zu Jeisten; denn natürlich sind auch der Anpassungsfähigkeit des gesunden Herzens gewisse Grenzen gesteckt ${ }^{2}$ ). Sowie aber des Stubenhockers Herz durch ein systematisches Trainiren in einigen Monaten sicherlich dazu gebracht werden kann, den oben genannten Anforderungen zu genügen, so bedingt die Schwangerschaft mit ihren ganz allmählich wachsenden Anforderungen eine fortschreitende Kräftigung des Herzmuske]s, die auch in einer objectiv feststellbaren Massenzunahme - proportional der Massenzunahme des Körpers - ihren Ansdruck findet. Wir dürfen aber noch weiter gehen: Diese Massenzunahme ist bereits sichtbarer Ausdruck dafür, dass das Herz auf die durch die Schwangerschaft geforderte Mehrleistung als auf eine mittlere Arbeitsleistung sich eingestellt hat. Somit haben wir die unter der Geburt gestellten Ansprüche nicht als Mehrarbeit nach dem Maasse der nichtgraviden Frau zu messen, sondern nur nach der Differenz gegenüber dem Ende der Schwangerschaft. Am Ende der Schwangerschaft ist ein neuer Ausgleich geschaffen; denn dieAccommodation erfolgtan sichschon unterErhöhung der Herzarbeit. Es würde zu weit führen, die experimentellen Grundlagen unserer Erkenntniss vom Accommodationsmechanismus hier zu erörtern. Ich verweise aber ausdrücklich auf die klassische Darstellung in Krehl's pathologischer Physiologie.

1) v. Frey, Deutsches Arch. f. klin. Med. Bd. 46. S. 398.

2) Krehl, Pathol. Physiologie. 3. Aufl. Leipzig. 1909. S. 4. 
Jaschke, Schwangerschaft, Geburt und Wochenbett bei Herzfehlern. 503

Etwas Wichtiges kommt noch hinzu: Diese durch die Schwangerschaft bedingte Erstarkung des Herzens ist mit einer Vergrösserung der Accommodationsbreite verbunden, d. h. das Herz wird jetzt wesentlich grössere Lieistungen als mittlere vollbringen können als vorher. Thatsächlich stehen ja unsere Erfahrungen mit diesen Folgerungen durchaus im Einklang. Die sogenannte Schwangerschaftshypertrophie des Herzens besteht in nichts anderem, als in einer der allgemeinen Zunahme der Körpermasse proportionalen Massenzunahme ${ }^{1}$ ). Das ist heute eine durchaus entschiedene Frage, der gegenüber entgegengesetzte Behauptungen endlich ganz verstummen sollten.

Diese allgemeinen physiologischen Bemerkungen müssen genügen. Ich denke, dass auf Grund derselben auch das Wesentliche des Accommodationsvorganges unter den uns interessirenden pathologischen Verhältnissen leicht einsehbar sind. Für alle Specialfälle der verschiedenen Herzfehler hier das pathologischphysiologische Geschehen zu entwickeln, würde zu weit führen und über den Titel meiner Arbeit hinausgehen. Das soll bei anderer Gelegenheit geschehen. Hier können nur noch einige allgemeine Gesetze vorgebracht werden, die mit unserem enger begrenzten Thema in näherer Beziehung stehen.

Zunächst sei wieder auf die Bedeutung der vorhergehenden Schwangerschaft verwiesen. Denn innerhalb weiter Grenzen darf eine erhöhte Anforderung an das Herz um so grösser sein, je langsamer sie sich entwickelt. Das Wesentlichste aber ist der Zustand des Herzmuskels, worauf ich schon wiederholt bei einzelnen Fällen hingewiesen habe. Freilich liegt hier auch der kritischste Punkt. Denn nicht so selten haben dieselben Vorgänge, die zur Entstehung des Klappenfehlers führten, auch den Herzmuskel geschädigt. Darnach kommt also Alles darauf an, ob zur Zeit des Eintritts der Schwangerschaft noch eine genügende Zahl von Muskelfasern intact ist, damit eine Anpassung an die erhöhten Anforderungen eintreten kann. Auf Grund unserer Erfahrungen dürfen wir annehmen, dass das in der Mehrzahl der Fälle zutrifft und gewöhnlich für eine ganze Reihe von Schwangerschaften zutrifft. Nur betrifft hier die Accommodation an die er-

1) W. Müller, Die Massenverhältnisse des menschlichen Herzens. Hamburg. 1884. S. 217 ff. - Dreysel, Ueber die Herzhypertrophie bei Schwangeren und Wöchnerinnen. München, 1891. München. med. Abhandlungen I. - Hirsch, Deutsch. Arch. f. klin. Med. Bd.64. S. 597. 
504 Jaschke, Schwangerschaft, Geburt und Wochenbett bei Herzfehlern.

höhten Anforderungen der Schwangerschaft und weiterhin der Geburt einen durch accommodative Hypertrophie vergrösserten Herzmuskel; ohne solche wäre ja die Compensation des Klappenfehlers unmöglich. Andererseits haben wir ein Recht anzunehmen, dass der hypertrophische Herzmuskel dieselbe Reservekraft besitzt, wie der gesunde!). Nur eine einzige Einschränkung kann praktisch wichtig werden, auf die wir schon oben hingewiesen haben. So könnte ja für manche Fälle eines Versagens des Herzens bei Mehrgebärenden an eine progressive Myocarditis im Sinne Albrecht's ${ }^{2}$ ) gedacht werden; indessen fehlen uns dafür zwingende Beweise und liegt meist eine andere Erklärung (Uebergreifen endarteriitischer Processe auf die Herzarterien, Erkrankung der Papillarmuskeln) näher. Kommt es einmal schon bei einer I p. zum Versagen der Accommodation an die erhöhten Ansprüche der Schwangerschaft, dann dürfen wir das wohl mit Recht auf mit dem Entstehen des Klappenfehlers synchrone myocarditische Veränderungen beziehen ${ }^{3}$ ). Von dem Grade dieser Veränderungen hängt es ab, ob es schon in der Schwangerschaft oder erst bei der Geburt zu einem Versagen kommt. Die von manchen Autoren, besonders von v. Guérard ${ }^{4}$ ) betonte Schädigung durch vorangegangene Geburten betrifft zum kleinsten Theil solche Herzen, vielmehr hängt das schliessliche Versagen oder die ungenügende Accommodation nach einer grösseren Reibe vorhergegangener Schwangerschaften und Geburten mit der kürzeren Lebensdauer des hypertrophischen Herzens im Allgemeinen zusammen. Unsere Fälle beweisen das schlagend, beweisen aber ebenso, dass diese Lebensdauer immerhin noch eine recht beträchtliche ist und oft genug erst im fünften Lebensdecennium ein Nachlass eintritt. Ich vermag also die Befürchtungen derjenigen Autoren, die Herzfebler für eine so ungünstige Complication der Schwangerschaft erklären, weder nach meinen praktischen Erfahrungen noch auf Grund experimenteller Thatsachen und theoretischer Ueberlegungen zu theilen.

Es bleibt noch eine praktisch sehr wichtige Frage zu entscheiden. Wir haben einmal überhaupt festzustellen, wie Frauen mit Herzfehlern unter der Geburt nothwendige operative Ein-

1) Krebl, l. c. S. $46 \mathrm{f}$.

2) Albrecht, Der Herzmuskel. II. Theil. Berlin 1903.

3) Albrecht, 1. c. - KrehI, Arch. f. klin. Med. 46. S. 454.

4) r. Guérard, l. c. 
Jaschke, Schwangerschaft, Geburt und Wochenbett bei Herzfehlern. 505 griffe, die ja fast immer unter Narkose ausgeführt werden müssen, ertragen, ferner aber, ob durch die Complication mit dem Herzfehler etwa eine Indication zu operativem Vorgehen unter der Geburt gegeben ist. Die eine Fehl- oder Frühgeburt einleitenden Eingriffe scheiden wir dabei aus, da wir schon oben, als wir die Indicationsstellung zur Schwangerschaftsunterbrechung besprachen, alles Wichtige angeführt haben. Ueber die Methoden der Einleitung der Frühgeburt können wir uns auch sehr kurz fassen, da von anderen Autoren diese Fragen genügend erörtert sind. Nur soviel: den Blasenstich empfehlen wir nicht, sondern nur die Bougiemethode oder die Metreuryse.

Eine Zusammenstellung der ausserdem vorgenommenen operativen Eingriffe ergiebt 52 operative Eingriffe und zwar:

10 mal typischen Forceps, darunter $1 \mathrm{mal}$ wegen Tympania uteri bei Insuff. valv, mitr. et aortae und Myodegeneratio.

1 hohen Forceps bei engem Becken.

8 "Manualhülfe bei Steisslagen; darunter sind ein Fall von Aortenstenose, ein Fall von decompensirter Insufficienz und Stenose der Mitralis.

4 "Wendung bei Querlage.

10 Wendung und Extraction. Darunter einmal, um bei drohender Gefahr von Seite des Herzens die Geburt zu beendigen. Exitus (Fall 650, cf., S. $475 \mathrm{ff}$.), einmal wegen drohenden Lungenödems. - Einmal kam es bei einer Wendung bei Hydramnios (uncomplicirte Mitralinsufficienz) zum Collaps, der aber rasch behoben werden konnte.

2 Kraniotomie wegen engen Beckens.

$1 "$ Decapitation.

5 "Wendung nach Braxton Hicks mit nachfolgendem Zug. Nur einmal war die Indication durch Herzschwäche und beginnendes Lungenödem gegeben bei Insuff. mitr. et aort. - Zweimal wurde die Operation wegen Eklampsie gemacht, einmal wegen Placenta praevia.

2 "Placentalösung; darunter 1 Exitus an Puerperalfieber (ef. Fall 415, S. 475).

3 "Hystrearyse zur Geburtsbeschleunigung; einmal wegen drohender Eklampsie. 
506 Jaschke, Schwangerschaft, Geburt und Wochenbett bei Herzfeblern.

1 mal Hysterotomia auterior (cf. Fall 605, S. 474).

1 "Sectio caesarea in mortua (cf. Fall 405, S. 475).

$1 "$ Porrooperation wegen Osteomalacie.

$5 "$ ist aus den Angaben der Frau nicht zu ersehen, was für Operationen gemacht wurden.

Nur 10 mal war unter der Geburt durch bedrohliche Erscheinungen von Seite des Herzens eine Indication zu einem Eingriff gegeben. Von den Todesfällen steht keiner mit einer Operation in unmittelbarem Zusammenhang.

Das Resultat dieser Nachforschung ist also, dass nur in 0,6 pCt. der Fälle durch den Herzfehler eine Indication zu einem Eingriff unter der Geburt gegeben ist. Nur zweimal kam es durch denselben zu bedrohlichen Erscheinungen, in allen anderen Fällen wurde sowohl der Eingriff selbst als die Narkose gut ertragen. Daraus ergeben sich für die Geburtsleitung wichtige Grundsätze. Aus der Veberschätzung der Gefahren hat sich vielfach eine ganz unberechtigte Polypragmasie entwickelt. Die meisten Autoren rathen dringend zu einer Abkürzung der Austreibungsperiode auf operativem Wege. Ich führe nur an, dass von v. Guérard forcirte Wendung und Extraction angerathen wird, Lvoff ${ }^{1}$ ) rühmt - wohl sehr mit Unrecht - die Expression nach Kristeller und selbst Olshausen ${ }^{2}$ ) hat seiner Zeit empfohlen, vor Beginn der Presswehen den Forceps anzulegen. Letzterer Vorschlag dürfte wohl das empfehlenswertheste Vorgehen sein in allen Fällen, in denen eive Abkürzung der Geburt durch drohende Herzschwäche erwünscht erscheint. Sonst aber möchten wir nach den hier mitgetheilten Erfahrungen der Ansicht Raum geben, dass auch die Complication mit Herzfehler an sich keinen Grund zu operativem Vorgehen unter der Geburt giebt. Wir stimmen Meyer-Ruegg ${ }^{3}$ ) ganz bei: "Es darf nicht zum Dogma werden, dass Herzfehler eine künstliche Abkürzung der Austreibungszeit durchaus bedingen... Das so gefürchtete Mitpressen wirkt auch bei hochgradiger Dyspnoe meist nicht verschlechternd auf den Zustand der Gebärenden; in wirklich bedrohlichen Lagen fällt es von selbst weg."

1) J. Lroff, Maladies du coeur et grossesse. Ann. de Gyn. et d'Obst. Tome XLVIIL. 1897. p. 489.

2) Olshausen,

3) Meyer-Ruegg, v, Winckel's Handbuch. Bd. Il. 3. Theil. 
Jaschke, Schwangersohaft, Geburt und Wochenbett bei Herzfehlern. 507

Andererseits scheint mir durch den Herzfehler kein einziger, aus anderen Gründen unter der Geburt nothwendiger Eingriff contraindicirt. Entgegen Fellner und Meyer-Ruegg, glaube ich, dass auch der Kaiserschnitt in keiner Weise Gefahren bringt. Die durch die rasche Entleerung des Uterus bedingte Druckschwankung fällt ja bei dem schon vorher eröffneten Abdomen verschwindend gering aus; und dass die Eröffnung der Bauchböhle gut vertragen wird, wissen wir doch aus so vielen Operationen an Herzkranken. Allerdings würde ich in dem vorliegenden Fall sehr rathen, den Kaiserschnitt lieber in Lumbalanästhesie auszuführen. Bei allen anderen entbindenden Operationen wird man wohl in der Lage sein, den Durehtritt des Kindes langsam zu bewerkstelligen. Nach der Geburt empfiehlt sich ein Sandsack auf den Leib als ebenso einfaches wie zuverlässiges Mittel.

Einer Operation möchten wir noch gedenken: der operativen Sterilisirung. Sie ist, glaube ich, angezeigt in allen Fällen, bei denen eine wesentliche Verschlimmerung des Herzleidens durch die Anstrengungen vorhergegangener Schwangerschaften und Geburten objectiv nachweisbar ist, und in einer späteren Gravidität bald Decompensation eintritt. Nicht durch die Einleitung des Abortus oder der Frühgeburt ist solchen Frauen zu helfen, sondern nur durch die Verhinderung jeder weiteren Conception. Handelt es sich um eine Frau am Ende der Gravidität, dann kann dieselbe mit dem Kaiserschnitt verbunden werden. Es ist nicht zu befürchten, dass diese Indication zur Sterilisirung $z u$ oft eintritt. Eine Durchsicht der Fälle, in denen die Schwangerschaftsunterbrechung indicirt war, ergiebt leicht, dass solche Fälle sehr in der Minderzahl sind.

\section{Ad VII.}

Von 4 Todesfällen (Fall 415, 571, 605 und 678 cf. S. 472-476) stehen wohl zwei (Fall 605 und 415) nicht mit dem Herzfehler in Zusammenhang.

Sonst finde ich 3 mal Thrombosen der Saphena notirt, die sämmtlich geheilt entlassen wurden. Zwei Fälle leichten Wochenbettfiebers zeigten gar keine Störungen von Seite des Herzens, in 4 Fällen schweren Puerperalfiebers ist einmal angegeben, dass von Seite des Herzens dabei starke subjective Beschwerden auftraten, 
508 Jaschke, Schwangerschaft, Geburt und Wochenbett bei Herzfehlern.

jedoch ohne objectiv nachweisbare bedrohliche Symptome, einmal kam es dabei zu einer recurrirenden Endocarditis. In einigen Fällen bestanden nach der Geburt die subjectiven Beschwerden noch einige Zeit fort, viermal ist eine auffallend hohe Pulsfrequenz (92-140) aufgefallen. Bemerkenswerth ist in einem Falle decompensirter Mitralinsufficienz das Auftreten wiederbolter kleinerer Nachblutungen, ohne dass in einem zurückgebliebenen Placentarrest die Ursache zu finden war.

Ich vermag allerdings über die Genauigkeit der Puls- und Herzcontrole in allen genannten Fällen kein Urtheil abzugeben. Wir gehen aber nicht fehl, aus den vorliegenden Daten den Schluss zu ziehen, dass schwerere Störungen im Wochenbett ausser in den angeführten wenigen Fällen jedenfalls nicht vorhanden waren. In allen Fällen also, in denen die Geburt gut abgelaufen ist, das sind $98 \mathrm{pCt}$. der Fälle, brauchen wir Störungen im Wochenbett im allgemeinen nicht zu fürchten. Ich vermag auch theoretisch die Gefahren des Wochenbetts bei Herzfehlern gar nicht einzusehen, trotzdem viele Autoren das Wochenbett fast mehr zu fürchten scheinen als die Geburt. Sobald das Kind ausgestossen ist, tritt zweifellos eine wesentliche Arbeitsverminderung für das Herz ein, es vermag sich zu erholen. Ein einziger Befund muss zunächst stutzig machen, die häufige Angabe fettiger Degeneration im Herzmuskel, die man mit der Involution direct in Zusammenhang gebracht hat. Worauf stützen sich aber diese Befunde? Ueberwiegend auf Sectionen von Wöchnerinnen, die an intercurrenten andern Krankheiten zu Grunde gegangen sind. Dass bei Sectionen von Frauen mit Herzfehlern, die unter der Geburt oder im Wochenbett starben, höhere Grade dieser Veränderungen sich finden, ist nur Beweis dafür, dass der Herzmuskel miterkrankt und sein Versagen die , wesentlichste Todesursache war. Das gilt aber, wie erwähnt, für eine verschwindende Minderzahl von Fällen. Für die übrigen Angaben scheint mir noch nicht einmal sicher gestellt, ob sie überhaupt mit dem Wochenbett etwas zu thun haben, noch weniger, ob ihnen die bedeutsame Rolle, die man ihnen zuschreibt, wirklich zukommt. Man findet diese Veränderungen, geringe und mittlere Grade fettiger, auch wohl körniger Degeneration, bei allen möglichen Zuständen. Was sie zu bedeuten haben, ist bis heute unklar. "Jedenfalls kommen sie aber gleichmässig bei functionstüchtigen und bei schwachen Herzen vor." (Krehl.) Und der braunen Atrophie des Herzmuskels 
Jaschke, Schwangerschaft, Geburt und Wochenbett bei Herzfehlern. 509

kommt nach heutiger Ansicht eine pathogenetische Bedeutung überhaupt kaum zu. ${ }^{1}$ )

Wichtig scheint zur. Verhütung von Gefahr lediglich eine entsprechende Wochenbettsdiätetik. Ruhe und sorgfältige, dem $\mathrm{Zu}-$ stand des Herzens angepasste Ernährung werden hier besondere Aufmerksamkeit erfordern. Das Frühaufstehen ist bei diesen Frauen zu vermeiden. - Endlich kann die Asepsis unter der der Geburt zu einem ausschlaggebenden Factor für die Prognose des Wochenbettes werden. Denn in einem bereits kranken Herzen besteht im Falle einer Infection immer die Gefahr der recurrirenden Endocarditis, die bei entsprechender Virulenz der Infection wohl ausschliesslich zum Tode führt. Dass bei guter Asepsis auch diese Gefahr wegfällt, versteht sich von selbst.

\section{Ad VIII.}

Ueber den Einfluss der Art des Herzfehlers ist nur wenig zu sagen. Aus unseren Fällen lässt sich ein besonderer Einfluss in dieser Richtung nicht feststellen. Alle die Fälle, wo man geneigt sein könnte, das Hauptgewicht auf die besondere Localisation des Vitiums zu legen, zeigen Complicationen und Besonderheiten, die die Deutung erschweren. Trotzdem sind die einzelnen Herzfehler nicht gleichwerthig. Es gilt für jeden auch bezüglich seines Einflusses auf Schwangerschaft, Geburt und Wochenbett genau dasselbe, was allgemein über die Prognose, über Unterschiede des Grades, der Art, der Entstehung eines Herzfehlers sich sagen lässt. Mehreres habe ich schon bei den einzelnen Fällen angedeutet. Eine Ausführung dieser Fragen gehört nicht in den Rahmen dieser Arbeit. ${ }^{2}$ )

\section{Zusammenfassung.}

Gestützt auf das grösste, bisher einheitlich geleitete und verfolgte Material gelangen wir durchwegs zu einer viel günstigeren Beurtheilung der Complication von Herzfehlern mit Schwangerschaft als fast alle anderen Autoren. Die hervorstechendste Thatsache

1) Krebl, Pathol. Physiologie. S. 61 f. - Ferner: Herzmuskelerkrankungen. S. 103. - Hasenfeld und v. Fenyvessy, Berliner klin. Wochenschrift. 1899. No. 4.

2) Näheres in den bereits genannten Werken von Jürgensen, Krebl, Romberg, fernerbei y.Leyden: Deutsche Klinik am Eingang des XX. Jabrhunderts. 
510 Jaschke, Schwangerschaft, Geburt und Wochenbett bei Herzfehlern.

unserer Untersuchungen ist die, dass ein Herzfehler in der Schwangerschaft nicht anders $z u$ beurtheilen sei, als ausserhalb derselben.

Wir finden unter 1525 Geburten bei herzfehlerkranken Frauen nur eine Mortalität von $0,39 \mathrm{pCt}$. bezw. $0,32 \mathrm{pCt}$. Dabei lässt sich auch für die überwiegende Mehrzahl der 'Todeställe feststellen, dass es sich um ganz besonders schwere Klappenfehler oder eine Combination mit Herzmuskelerkrankung handelte. Kurz, diese Fälle zeigen in verschiedener Hinsicht zum grossen Theil eine so ausgesprochene Sonderstellung, dass sie dadurch an Bedeutung als Beispiele für die allgemeine Beeinflussung der Herzfehler durch die Schwangerschaft und Geburt einbüssen. - Unserer Zahl gegenüber erscheinen die Angaben vieler anderer Autoren ${ }^{1}$ ) über eine Mortalität von 12-60-100 pCt. ganz enorm, was wir damit glauben erklären zu können, dass deren Fälle zum Theil besonders schwere waren und die Berechnung sich oft nur auf ein ganz kleines Material (selten über 20 Fälle) stützt. Anch die von Fellner (l. c.) aus der Gesammtliteratur unter Einbeziehung seiner Fälle berechnete Mortalität von $7,3 \mathrm{pCt}$. ist noch viel zu hoch gegriffen. Dagegen fällt Fellner's aus seinen eigenen Beobachtungen berechnete Zahl von 1,3 pCt. Mortalität mit unseren Erfahrungen ungefähr zusammen, wenn man berücksichtigt, dass Fellner selbst meint, nur etwa $1 / 7$ der Herzfehler thatsächlich berücksichtigt zu haben, während $6 / 7$, die leichteren Fälle, übersehen worden sein mögen.

Nach Fellner und Schauta tritt in 20,2 pCt. eine spontane Unterbrechung der Schwangerschaft ein, sei es als Fehloder als Frühgeburt. Andere Autoren haben zum Theil noch höhere Zahlen angegeben. Aus unseren Feststellungen dagegen ergiebt sich, dass in $7 / 8$ der Fälle eine spontane Geburt am Ende der Gravidität erwartet werden kann. Nur in etwa 4 pCt. ist ein Spontanabort, in etwa 4,5 pCt. eine spontane Frühgeburt zu erwarten. Ein besonderer Einfluss der Art des Herzfehlers ist dabei nicht festzustellen. Decompensation begünstigt die vorzeitige Schwangerschaftsunterbrechung, das Alter und die Geburtenzahl sind jedoch in der gewöhnlichen Weise bei der Annahme eines causalen Zusammenhanges zwischen Herzfehler und vorzeitiger Schwangerschaftsunterbrechung mit in Rechnung zu

1) Näheres of. bei Fellner (l. c.), der die Literatur so vollständig verarbeitet hat, dass hier von einer Wiederholung abgesehen werden kann. 
Jaschke, Schwangerschaft, Geburt und Wochenbett bei Herzfehlern. 511

ziehen. - In manchen Fällen scheint umgekehrt eine Accoutumance aux cardiopathies im Sinne Baranger's anzunehmen.

Eine künstliche Unterbrechung der Schwangerschaft erweist sich nur in etwa 1 pCt. der Fälle als nothwendig. Die Indication dazu darf als gegeben erachtet werden durch schwere Decompensation. Bei leichteren Compensationsstörungen nur dann, wenn unter interner Therapie keine volle Leistungsfähigkeit eintritt; unter weiterer Berücksichtigung der Art des Herzfehlers, des Alters, der Geburtenzahl. Ob trotz wiedererlangter und längere Zeit erhaltener Compensation in einem bestimmten Falle eine Unterbrechung der Schwangerschaft, gewissermaassen prophylaktisch, berechtigt ist, wird unter Berücksichtigung aller anderen Umstände durch die Functionsprüfung des Herzens zu entscheiden sein. Die für uns zur Functionsprüfung geeigneten Methoden sind die von Katzenstein ${ }^{1}$ ), in Kliniken eventuell auch die von Korányi zuerst angegebene von Knecht und Ritoók ausgebaute Methode ${ }^{2}$ ).

Die Schwangerschaftsunterbrechung ist aber jedenfalls nur als ein momentanes Hilfsmittel anzusehen. Hat man in einem speciellen Falle aus dem Verlaufe früherer Schwangerschaften und Geburten die Ueberzeugung gewonnen, dass jede weitere Belastung des Herzens durch eine erneute Gravidität oder gar durch die Geburtsarbeit für die Frau lebensgefährlich sei, dann ist die operative Sterilisirung angezeigt.

Eine Gefährdung durch die Schwangerschaft gilt aber nur für wenige Fälle besonders schwerer Herzfehler oder der Miterkrankung des Herzmuskels, sowie für Fälle, die mit Nephritis

1) Das Princip der Methoden besteht in der vergleichenden Controle von Puls und Blutdruck vor, während und nach Ausschaltung des Gefässgebietes der Aa. femorales. Bei Ausschaltung psychischer Erregung giebt die Methode ganz brauchbare Resultate. Näheres muss in den Originalarbeiten von Katzen stein nachgelesen werden. (Zeitschr. f. klin. Med. Bd. 50. S. 322. 1903. Deutsche med. Wochenschr. 1904. No. 22 und 23. - Med. Klinik. 1906. S. 1035.)

2) Die Methode von Korányi (Zeitschr. f. klin. Med. Bd. 33 und 34) besteht in der Verfolgung des Quotienten $\frac{\Delta}{\mathrm{NaCl}}$ vor und nach körperlicher Bewegung. Näheres für die uns interessirenden Fälle cf. Knecht (Deutsches Arch. f. klin. Med. Bd. 83. S. 266. 1905) und Ritoók (Deutsche med. Wochenschr. 1909. No. 34). - Bei dieser Methode lässt sich die psychische Componente ganz ausschalten, für die tägliche Anwendung im Betrieb einer grösseren Klinik ist sio aber zu complicirt. 
512 Jaschke, Schwangerschaft, Geburt und Wochenbett bei Herzfehlern.

complicirt sind. In allen anderen Fällen (in $96 \mathrm{pCt}$.) kann man auf einen ungestörten Verlauf der Sehwangerschaft rechnen.

98 pCt. aller Geburten bei Herzfehlern verlaufen ohne Störung, selbst bei geringer Decompensation. Die oft behauptete Atoniegefahr ist an unserem Material nicht nachweisbar. Eine Indication zu operativem Eingreifen ist durch den Herzfehler an sich in keiner der drei Geburtsperioden gegeben. Aerztliche sorgfältige Ueberwachung der Geburt, besonders in der Austreibungsperiode ist aber nothwendig, um bei irgend bedrohlichen Symptomen sofort eingreifen zu können. Kein einziger, aus irgend welchen Gründen nothwendiger operativer Eingriff ist durch den Herzfehler contraindicirt.

Peinlichste Asepsis ist hier noch dringender als sonst geboten, da durch Puerperalfieber eine herzkranke Frau viel mehr gefährdet ist als eine herzgesunde, ceteris paribus. Davon abgesehen drohen der Herzfehlerkranken im Wochenbett nicht mehr Gefahren als der Gesunden. Längere Bettruhe, als einfachstes Mittel zur Herabsetzung der Herzarbeit und Erholung ist hier angezeigt. Das Stillen der Frau ist in keinem Falle contraindicirt, entgegen den Angaben mehrerer Autoren.

Ein besonderer Einfluss der Art des Herzfehlers auf den Verlauf der Schwangerschaft u. s. w. lässt sich im Allgemeinen nicht feststellen. Auch in dieser Hinsicht bestehen keine anderen Maasse für die Werthung eines Herzfehlers als ausserhalb der Gravidität. Die sich ergebenden Unterschiede sind einzig im Accommodationsmechanismus der verschiedenen Klappenfehler begründet. Jede Erkrankung des Herzmuskels, gleichgültig ob sie der Klappenerkrankung coordinirt ist oder erst später durch Infection, Intoxication oder das Uebergreifen endarteriitischer Processe von der Aorta auf die Aa. coronariae bedingt ist, trübt die an sich günstige Prognose. In dieser Hinsicht kommt dem Alter ein bedeutender Einfluss zu. In äbnlicher Weise ist der ungünstige Einfluss einer grösseren Zahl namentlich rasch auf einander folgender Schwangerschaften zu erklären; die Lebensdauer des hypertrophischen Herzens ist eine geringere. 
Name:Floder Anaz.

Joumal-1r.2925

Frankheit Itium de comp + Groom. WT

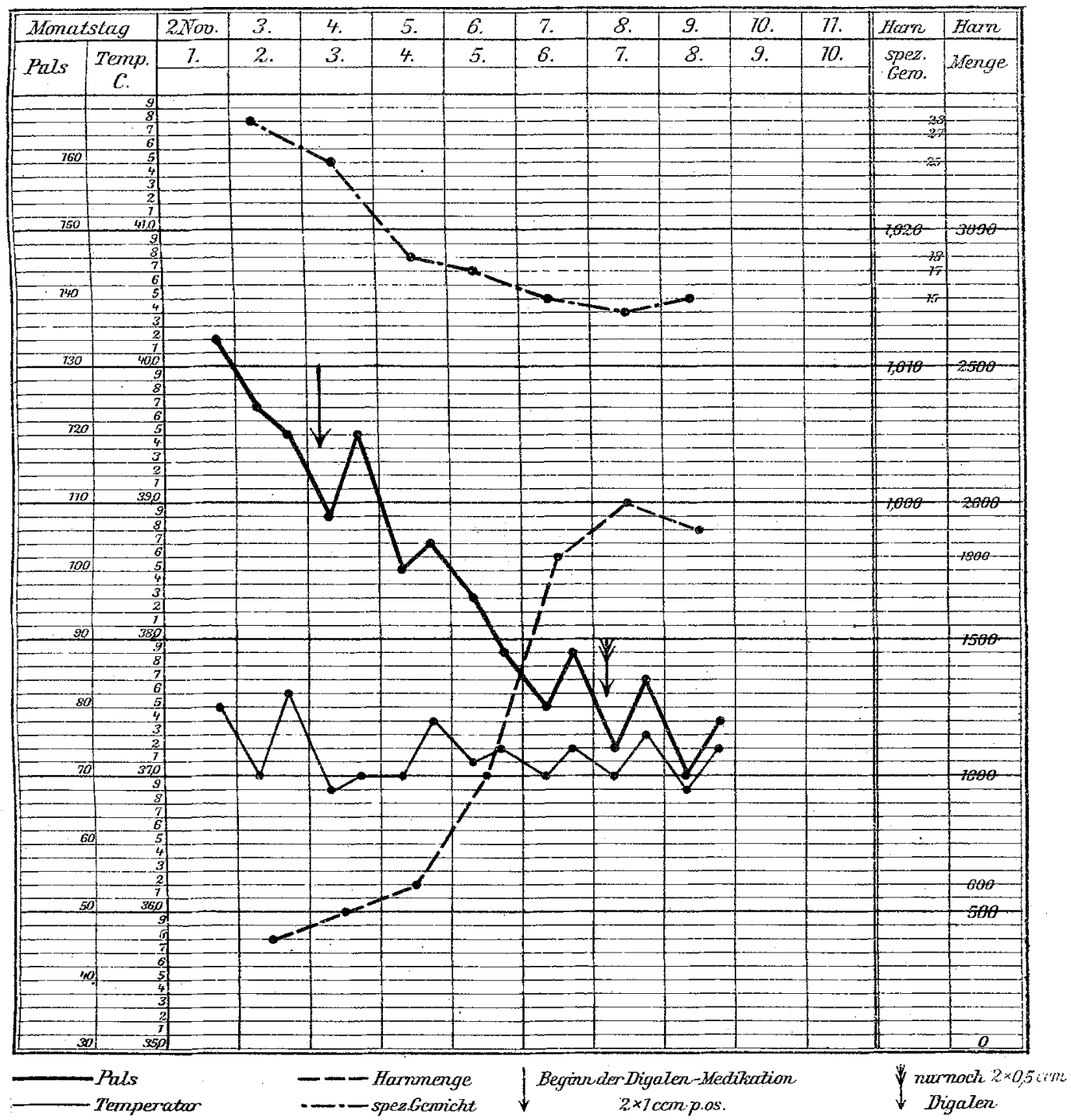

